ARTICLE

https://doi.org/10.1038/s41467-019-13806-x

\title{
Identification of recurrent FHL2-GL12 oncogenic fusion in sclerosing stromal tumors of the ovary
}

Sarah H. Kim¹,13, Arnaud Da Cruz Paula1,13, Thais Basili (10) 2,13, Higinio Dopeso (1) 2,13, Rui Bi 2,3,13, Fresia Pareja2, Edaise M. da Silva (1) 2 ${ }^{2}$, Rodrigo Gularte-Mérida (1) 1,2, Zhen Sun (1) 4, Sho Fujisawa (1) ${ }^{5}$, Caitlin G. Smith², Lorenzo Ferrando (10 2,6, Ana Paula Martins Sebastião 2,7 , Yonina Bykov ${ }^{8}$, Anqi Li ${ }^{2,3}$, Catarina Silveira (i) ${ }^{2,9}$, Charles W. Ashley', Anthe Stylianou', Pier Selenica ${ }^{2}$, Wesley R. Samore ${ }^{10}$, Achim A. Jungbluth², Dmitriy Zamarin ${ }^{8}$, Nadeem R. Abu-Rustum¹, Kristian Helin (1) 4,11,12, Robert A. Soslow², Jorge S. Reis-Filho (D) ${ }^{2}$, Esther Oliva ${ }^{10 \star} \&$ Britta Weigelt ${ }^{2 \star}$

Sclerosing stromal tumor (SST) of the ovary is a rare type of sex cord-stromal tumor (SCST), whose genetic underpinning is currently unknown. Here, using whole-exome, targeted capture and RNA-sequencing, we report recurrent FHL2-GLI2 fusion genes in 65\% (17/26) of SSTs and other GLI2 rearrangements in additional 15\% (4/26) SSTs, none of which are detected in other types of SCSTs $(n=48)$ or common cancer types $(n=9,950)$. The FHL2-GLI2 fusions result in transcriptomic activation of the Sonic Hedgehog ( $\mathrm{SHH}$ ) pathway in SSTs. Expression of the FHL2-GLI2 fusion in vitro leads to the acquisition of phenotypic characteristics of SSTs, increased proliferation, migration and colony formation, and $\mathrm{SHH}$ pathway activation. Targeted inhibition of the $\mathrm{SHH}$ pathway results in reversal of these oncogenic properties, indicating its role in the pathogenesis of SSTs. Our results demonstrate that the FHL2-GLI2 fusion is likely the oncogenic driver of SSTs, defining a genotypic-phenotypic correlation in ovarian neoplasms.

\footnotetext{
${ }^{1}$ Department of Surgery, Memorial Sloan Kettering Cancer Center, New York, NY 10065, USA. ${ }^{2}$ Department of Pathology, Memorial Sloan Kettering Cancer Center, New York, NY 10065, USA. ${ }^{3}$ Department of Pathology, Fudan University Shanghai Cancer Center, 200032 Shanghai, People's Republic of China. ${ }^{4}$ Cell Biology Program and Center for Epigenetics, Memorial Sloan Kettering Cancer Center, New York, NY 10065, USA. ${ }^{5}$ Molecular Cytology Core Facility, Memorial Sloan Kettering Cancer Center, New York, NY 10065, USA. ${ }^{6}$ Department of Internal Medicine, University of Genoa, 16126 Genova, Italy. ${ }^{7}$ Department of Medical Pathology, Federal University of Paraná, Curitiba, Paraná 80060-000, Brazil. ${ }^{8}$ Department of Medicine, Memorial Sloan Kettering Cancer Center, New York, NY 10065, USA. ${ }^{9}$ GenoMed SA, Institute of Molecular Medicine, University of Lisbon, 1649 Lisbon, Portugal. ${ }^{10}$ Department of Pathology, Massachusetts General Hospital, Harvard Medical School, Boston, MA 02114, USA. ${ }^{11}$ Biotech Research and Innovation Centre (BRIC), University of Copenhagen, 2200 Copenhagen, Denmark. ${ }^{12}$ The Novo Nordisk Foundation for Stem Cell Biology (Danstem), University of Copenhagen, 2200 Copenhagen, Denmark. ${ }^{13}$ These authors contributed equally: Sarah H. Kim, Arnaud Da Cruz Paula, Thais Basili, Higinio Dopeso, Rui Bi. *email: eoliva@mgh.harvard.edu; weigeltb@mskcc.org
} 
S ex cord-stromal tumors are a heterogeneous group of benign and malignant neoplasms derived from the primitive sex cords or stromal components of the ovary ${ }^{1}$. Sclerosing stromal tumors (SSTs) represent an uncommon subtype of sex cord-stromal tumor $(<5 \%)^{1-3}$, occurring in young adults in the second and third decades of life ${ }^{4}$. Patients typically present with abdominal or pelvic pain and menstrual irregularities in the setting of a unilateral pelvic mass. SSTs are usually hormonally inactive, however androgenic changes in the setting of pregnancy or virilization have been reported ${ }^{4}$. Although regarded as a benign neoplasm, its clinical presentation, imaging findings and elevated CA-125 levels may mimic those of malignant ovarian neoplasms, resulting in young women undergoing radical surgical resection ${ }^{2,5,6}$. In addition, the panoply of histologic features SSTs display may pose diagnostic challenges and result in misdiagnoses 2,7 . Immunohistochemistry may be helpful as these tumors are positive for sex cord markers including calretinin and FOXL $2^{8-10}$; however, understanding the genetic underpinning of SSTs may aide in the diagnosis and management of these rare ovarian neoplasms.

The genomic landscape of common types of ovarian cancer has been thoroughly investigated ${ }^{11}$, and the genetic underpinnings of some rare types of ovarian cancer have been identified. For instance, SMARCA4 loss-of-function mutations have been documented in small cell carcinomas of the ovary hypercalcemic type $^{12}$ and FOXL2 p.C134W hotspot mutations have been described in $>97 \%$ of adult-type granulosa cell tumors ${ }^{1,9}$, the most common sex cord-stromal tumor. These seminal studies indicate the vast potential for the discovery of unique genomic drivers in rare types of ovarian tumors ${ }^{13}$. In addition, DICER1 mutations have been detected in a subset of Sertoli-Leydig cell tumors and other non-epithelial ovarian cancers ${ }^{14,15}$. The genetic landscape of other sex cord-stromal tumors, including SSTs, however, is currently unknown.

We posited that if SSTs are driven by a pathognomonic genetic alteration, this information could be used for the development of ancillary markers to mitigate the diagnostic challenges posed by these rare tumors. In this study, we sought to define the repertoire of genetic alterations in SSTs, using a combination of wholeexome sequencing, targeted massively parallel sequencing and RNA-sequencing. Our analyses reveal the presence of a highly recurrent FHL2-GLI2 fusion transcript or GLI2 rearrangements in SSTs. Functional analyses in vitro establish that expression of the FHL2-GLI2 fusion increases signaling via the Sonic Hedgehog (SHH) pathway and results in the acquisition of oncogenic properties, which can be reversed through its chemical inhibition, thereby establishing a genotypic-phenotypic correlation and the importance of the SHH pathway in the biology of these tumors.

\section{Results}

Clinical and histologic features of SSTs. SSTs were retrieved from the authors' institutions, following approval by the institutional review boards (IRBs)/local ethics committees, and patient consents were obtained where appropriate. Following central pathology review, 26 tumors were classified as SSTs and included in this study (Supplementary Table 1, Supplementary Fig. 1). Patient median age at diagnosis was 29 (range 14-56) years, and all patients underwent surgical resection without any further adjuvant treatment (Supplementary Table 1). Histologically, SSTs were characterized by alternating areas of hypercellularity and hypocellularity imparting a vague lobulated architecture. An often prominent component of staghorn vessels, as well as varying numbers of spindle and luteinized stromal cells with overall bland cytologic features and overall low mitotic and proliferation rates were noted (Fig. 1a, Supplementary Table 1, Supplementary Fig. 2).
SSTs display few mutations and copy number alterations. To determine whether SSTs are underpinned by a pathognomonic somatic mutation, DNA samples from eight SSTs and matched normal tissues were subjected to whole-exome sequencing (WES; $n=3$, median depth of coverage of tumor $108 \times$, range $50 \times-226 \times$, and normal samples $22 \times$, range $20 \times-144 \times$ ) or to targeted massively parallel sequencing due to the limited yields of DNA available ( $n=5$, median depth of coverage of tumor samples $698 \times$, range $423 \times-855 \times$ ) using the Memorial Sloan Kettering-Integrated Mutation Profiling of Actionable Cancer Targets (MSK-IMPACT ${ }^{16}$ ) assay (Supplementary Fig. 1, Supplementary Table 1). We reasoned that if SSTs were to harbor a somatic mutation at the same frequency of FOXL2 mutations in adult-type granulosa cell tumors ${ }^{9}$, eight samples would confer $>90 \%$ of statistical power to detect a pathognomonic mutation.

SSTs displayed a low mutation burden, with a median of 16 (range 4-36) somatic mutations identified by WES, of which 9 (range 3-10) were non-synonymous. MSK-IMPACT sequencing detected a median of 2 (range 1-3) somatic mutations, with only 1 (range 0-3) being non-synonymous (Supplementary Table 2). None of the mutations identified were recurrent in the SSTs analyzed. In fact, the majority of mutations were non-pathogenic missense mutations not affecting hotspot residues, and three of the five SSTs subjected to MSK-IMPACT sequencing did not harbor any mutations affecting the 410 cancer-related genes tested (Supplementary Fig. 3). One potentially pathogenic nonsynonymous somatic mutation was identified, a subclonal ATR $\left(\mathrm{p} . \mathrm{R} 1183^{\star}\right.$ ) nonsense mutation in SST2 (Supplementary Fig. 3, Supplementary Table 2). Genome-wide copy number analysis revealed a paucity of copy number alterations, and the eight SSTs analyzed displayed diploid/near-diploid genomes. No homozygous deletions or amplifications were detected in any of the SSTs studied (Supplementary Fig. 3).

Taken together, SSTs are characterized by a low mutation burden, low levels of genomic instability, and the lack of recurrent somatic mutations or copy number alterations.

SSTs of the ovary harbor a recurrent FHL2-GLI2 fusion gene. RNA-sequencing of eight SSTs revealed a recurrent in-frame FHL2-GLI2 fusion gene with a high driver probability as defined by OncoFuse ${ }^{17}$ in $50 \%(4 / 8)$ of the cases analyzed (Supplementary Table 3). This fusion gene resulted in a chimeric transcript composed of exons 1-5 of FHL2 (chr2:105984027) and exons 8-13 of GLI2 (chr2:121732551), likely through a paracentric inversion of chromosome 2q (Fig. 1b). FHL2 (Four And A Half LIM Domains 2) is a member of the four and a half LIM domainonly family, which is constitutively expressed in ovarian tissues $^{18,19}$, whereas the oncogene GLI2 (GLI Family Zinc Finger 2) encodes a zinc finger transcription factor, which comprises a DNA binding domain, a GLI2 repression domain at the $\mathrm{N}$-terminus and an activation domain at its C-terminus ${ }^{20}$ (Fig. 1b). In this fusion, two of the four LIM protein domains of FHL2 were retained, while the repression domain of GLI2 was lost (Fig. 1b). The fusion of FHL2 to the 3 '-end of GLI2 resulted in increased expression of exons 9-13 of GLI2, including the DNA binding and activation domains (Fig. 1c). Based on the domains involved in the chimeric transcript and the patterns of exonic transcription in SSTs harboring the FHL2-GLI2 chimeric gene (Fig. 1c), the most parsimonious explanation is that the $5^{\prime}$ aspects of FHL2 are juxtaposed with the DNA binding and activation domains of GLI2, coupled with a loss of the GLI2 repression domain $18,19,21$. As important mediators of the SHH pathway ${ }^{22}$, the GLI family proteins, GLI1-3, play an integral role in embryogenesis and are regarded as potent oncogenes ${ }^{23,24}$. Specifically, GLI2 functions as a transcription factor binding DNA 
a

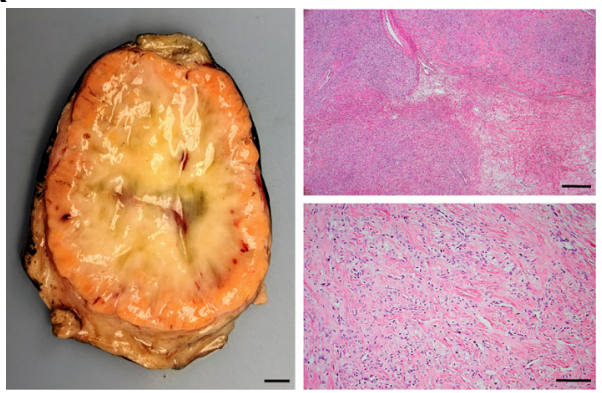

c

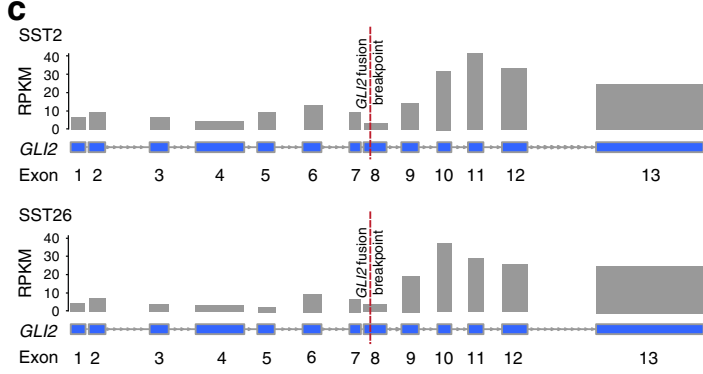

e

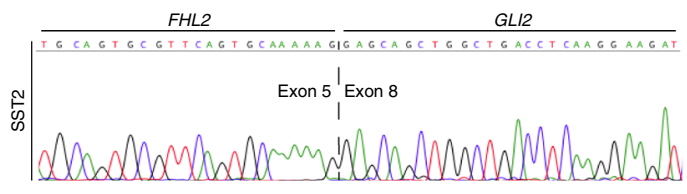
FHL2

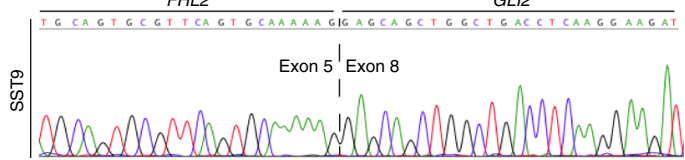

g

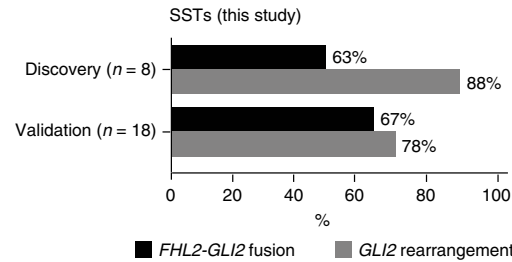

i

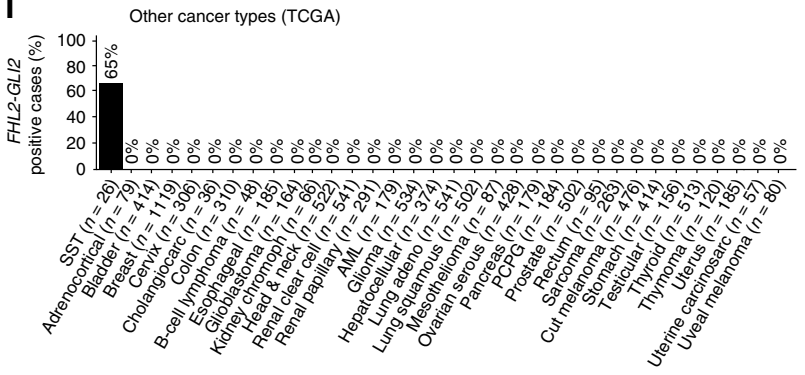

This b

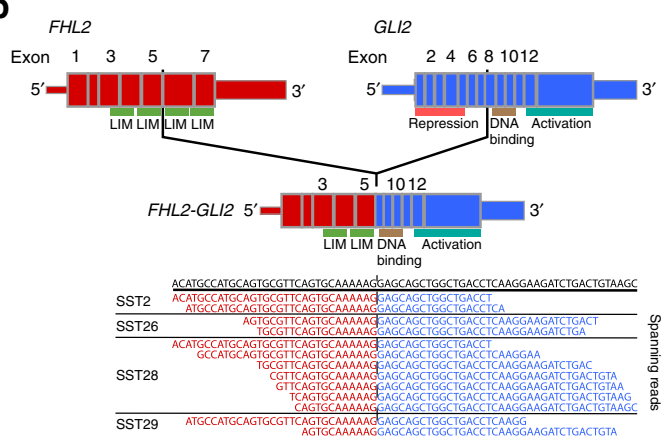

d
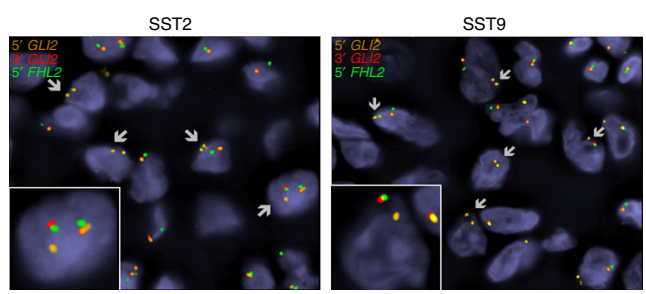

$\mathbf{f}$

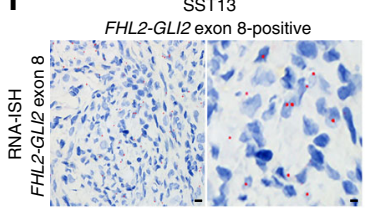

SST27
FHL2-GLI2 exon 7-positive
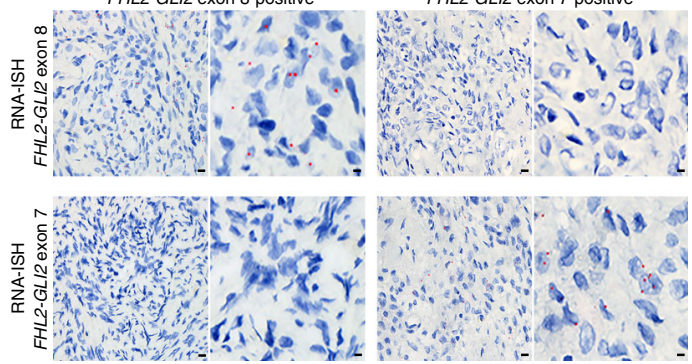

h

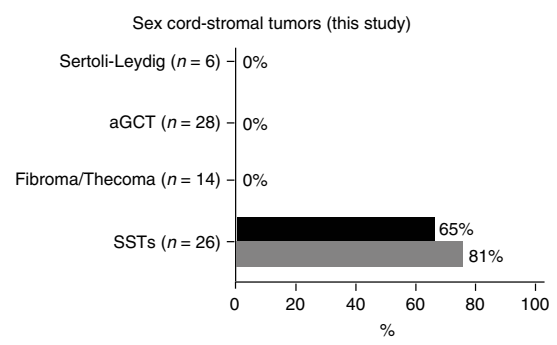

- FHL2-GL12 fusion through zinc finger motifs ${ }^{22,24}$, and GLI1 has been found to constitute an oncogenic partner in fusion genes described in gastroblastoma ${ }^{25}$, soft tissue sarcomas ${ }^{26}$, and fibromyxomas ${ }^{27}$.

One of the initial eight SSTs of the discovery cohort, SST27, harbored a FHL2-GLI2 fusion involving exons 7-13 of GLI2, rather than exons 8-13 of GLI2 found in the remaining 16 SSTs; this chimeric FHL2-GLI2 transcript in SST27 also leads to a juxtaposition of the $5^{\prime}$ elements of FHL2 with GLI2 lacking the repression domain whilst leaving the DNA binding and activation domains intact (Supplementary Fig. 4a, Supplementary Table 3). In addition, SST12, which lacked the FHL2-GLI2 fusion as assessed by RNA-sequencing and RT-PCR, was found to harbor a DYNLL1-GLI2 fusion gene with a high driver probability as defined by OncoFuse ${ }^{17}$ (Supplementary Table 3), where the 
Fig. 1 Recurrent FHL2-GLI2 fusion gene in sclerosing stromal tumors of the ovary. a Photograph of the cut section of an ovarian sclerosing stromal tumor (SST; left) displaying classic SST appearance with yellow tissue at periphery and white, central fibrotic depression, and micrographs of hematoxylin \& eosin stained representative section at low (top right) and high (bottom right) magnification. Scale bars, $1 \mathrm{~cm}$ (left), $200 \mu \mathrm{m}$ (top right), $50 \mu \mathrm{m}$ (bottom right). b Schematic representation of the FHL2-GLI2 fusion transcript including the exons and domains involved. The breakpoint of the $5^{\prime}$ and $3^{\prime}$ partner genes are represented as black vertical lines. Spanning reads are depicted and aligned to the predicted junction sequence. c Schematic representation showing the Reads Per Kilobase per Million (RPKM) mapped read counts of each GLI2 exon. The GLI2 fusion breakpoint is represented as a red dashed line. d Fluorescence in situ hybridization (FISH) of two representative SSTs using a three-color FHL2-GLI2 probe, with 5' GLI2 (orange), 3' GLI2 (red), and 5' FHL2 (green), showing the presence of the FHL2-GLI2 fusion (white arrows). e Representative Sanger sequencing electropherograms of the genomic FHL2GLI2 breakpoint. f RNA in situ hybridization (RNA-ISH) using custom FHL2-GLI2 probes (red) showing the chimeric FHL2-GLI2 mRNA expression in two representative SSTs harboring the FHL2-GLI2 fusion. g Frequency of the FHL2-GLI2 fusion gene and GLI2 rearrangements in 26 SSTs from this study. h Frequency of the FHL2-GLI2 fusion gene and GLI2 rearrangements in 26 SSTs and frequency of the FHL2-GLI2 fusion gene in 48 other ovarian sex cordstromal tumors from this study. aGCT, adult-type granulosa cell tumor. i Frequency of FHL2-GLI2 fusion gene in 26 SSTs from this study and in 9950 tumors from 33 cancer types from The Cancer Genome Atlas (TCGA). AML acute myeloid leukemia, PCPG pheochromocytoma and paraganglioma.

chimeric transcript was composed of exons 1-2 of DYNLL1 and exons 8-13 of GLI2 (Supplementary Fig. 4b).

To validate the presence of the GLI2 fusion genes found by RNA-sequencing, we performed reverse transcription (RT)-PCR (for FHL2-GLI2 exons 7-13 GLI2, exons 8-13 GLI2, and DYNLL1GLI2) and/or fluorescence in situ hybridization (FISH) analysis with custom GLI2 break-apart probes in the initial eight SSTs and an additional 18 SSTs (validation cohort), for which RNA of sufficient quantity/quality for RNA-sequencing could not be obtained. The presence of the FHL2-GLI2 fusion gene was validated in the index cases, and 12 of the additional 18 SSTs (67\%) were found to harbor an FHL2-GLI2 fusion transcript (Figs. 1d-f, Supplementary Table 4). The only recurrent GLI2 fusion gene identified in the 26 cases tested was the FHL2-GLI2 comprising exons 8-13 of GLI2 (Fig. 1a, b); the FHL2-GLI2 containing exons 7-13 GLI2 and DYNLL1-GLI2 fusions were found to be restricted to SST27 and SST12, respectively (Supplementary Fig. 4). FISH analysis also revealed the presence of break-apart signals of the 5'GLI2-3'GLI2 probe in 3 SSTs lacking FHL2-GLI2 and DYNLL1-GLI2 fusions by RNA-sequencing and/or RT-PCR (SST1, SST8, SST20), indicative of a GLI2 rearrangement (Supplementary Fig. 5, Supplementary Table 4).

In total, $21 / 26(81 \%)$ of the SSTs analyzed harbored GLI2 rearrangements, with $16 / 26$ (62\%) harboring the FHL2GLI2 comprising exons $8-13$ of GLI2, 1/26 (4\%) having a FHL2-GLI2 containing exons 7-13 GLI2, 1/26 (4\%) displaying a DYNLL1-GLI2 fusion gene and 3/26 (12\%) harboring other forms of GLI2 rearrangements whose partners have yet to be identified (Fig. 1g). Of note, no differences in the histologic or clinical features of the SSTs according to the presence/absence of the GLI2 rearrangements, the type of GLI2 rearrangements or the partner of the GLI2 rearrangements were observed (Supplementary Table 1). Reanalysis of the whole-exome and targeted capture sequencing data of the GLI2 wild-type SSTs failed to reveal any likely driver genetic alteration.

FHL2-GLI2 fusion gene as a defining feature of ovarian SSTs. To determine whether the FHL2-GLI2 fusion gene would constitute a pathognomonic genetic alteration for SSTs, we investigated the presence of the FHL2-GLI2 and the DYNLL1-GLI2 fusions in other sex cord-stromal tumors by RT-PCR and FISH, and across 33 common cancer types from The Cancer Genome Atlas (TCGA) retrieved from the Tumor Fusion Gene Data Portal $^{28}$. Whilst the FHL2-GLI2 and DYNLL1-GLI2 rearrangements were present in $17 / 26(65 \%)$ and $1 / 26(4 \%)$ of SSTs, respectively, none of the other ovarian sex cord-stromal tumors tested by RT-PCR, namely adult-type granulosa cell tumors $(n=$ $28)$, fibromas, fibrothecomas and thecomas $(n=14)$ and SertoliLeydig cell tumors (SLCTs, $n=6$ ), and none of the 9950 tumors of 33 cancer types from TCGA ${ }^{28}$ harbored the FHL2-GLI2 or
DYNLL1-GLI2 fusion genes (Fig. 1h, i, Supplementary Fig. 4, Supplementary Table 4). In addition, no GLI2 break-apart signals/rearrangements were found using FISH in other sex cordstromal tumors $(n=9$; Supplementary Fig. 5, Supplementary Table 4). Taken together, these findings provide evidence to suggest that FHL2-GLI2 and DYNLL1-GLI2 fusions might be pathognomonic for SSTs of the ovary.

Consistent with the RNA-sequencing and RT-PCR findings, RNA in situ hybridization (RNA-ISH) demonstrated mRNA expression of the specific forms of the chimeric FHL2-GLI2 fusion gene in the SSTs harboring each of the two FHL2-GLI2 fusion genes (Fig. 1f, Supplementary Fig. 6, Supplementary Table 4). In addition, immunohistochemistry with anti-GLI2 antibodies revealed moderate to high levels of GLI2 protein expression in SSTs with and without the FHL2-GLI2 fusion (Supplementary Figs. 2 and 4), respectively, supporting the notion that SSTs may be a disease driven by activation of GLI2.

FHL2-GLI2 expression results in oncogenic properties. Based on the known biological roles of FHL2 and GLI2, we hypothesized that cells expressing the FHL2-GLI2 fusion would exhibit oncogenic behavior through overexpression of the DNA binding and activation domains of GLI2 coupled with the loss of its repression domain. The FHL2-GLI2 fusion gene encompassing exons 8-13 of GLI2, recurrently detected in SSTs, as well as truncated ( $\mathrm{t})$ GLI2 lacking the repression domain were evaluated in multiple cell models, including immortalized mesenchymal stem cells (MSCs), HEK-293 cells, and in cells derived from cancers with known deregulation of the SHH pathway $22,29,30$, namely medulloblastoma (DAOY) and basal cell carcinoma (BCC) cells. MSC, HEK-293, DAOY, and BCC cells stably expressing tGLI2 or the FHL2-GLI2 fusion displayed a significant increase in proliferation and colony formation as compared to the same cells expressing empty vector, wild-type FHL2, or wild-type GLI2 (Fig. 2a, b). In addition, a scratch assay analysis revealed increased migration of MSCs, HEK-293, DAOY, and BCC cells upon stable expression of tGLI2 and FHL2-GLI2 as compared to cells expressing empty vector, wild-type FHL2 or wild-type GLI2 (Fig. 2c). Akin to the partners involved in oncogenic fusion genes, an increase in proliferation, colony formation and migration in cells transduced with wild-type GLI2 was observed, however, cells harboring tGLI2 or the FHL2-GLI2 fusion consistently significantly exhibited a more overt phenotype (Fig. 2a-c).

FHL2-GLI2 expression results in SST phenotypic features. We next sought to define whether the expression of the FHL2-GLI2 fusion would result in the acquisition of phenotypic features of SSTs in the cell models tested. Calretinin, a calcium-binding protein encoded by the CALB2 gene, is primarily expressed in 
a

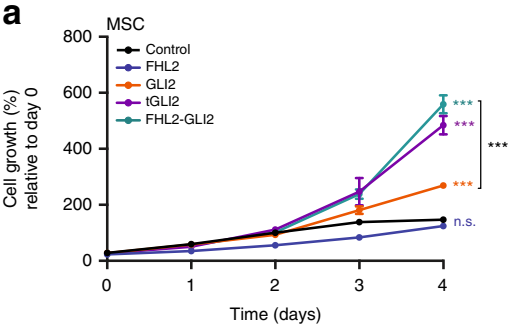

b
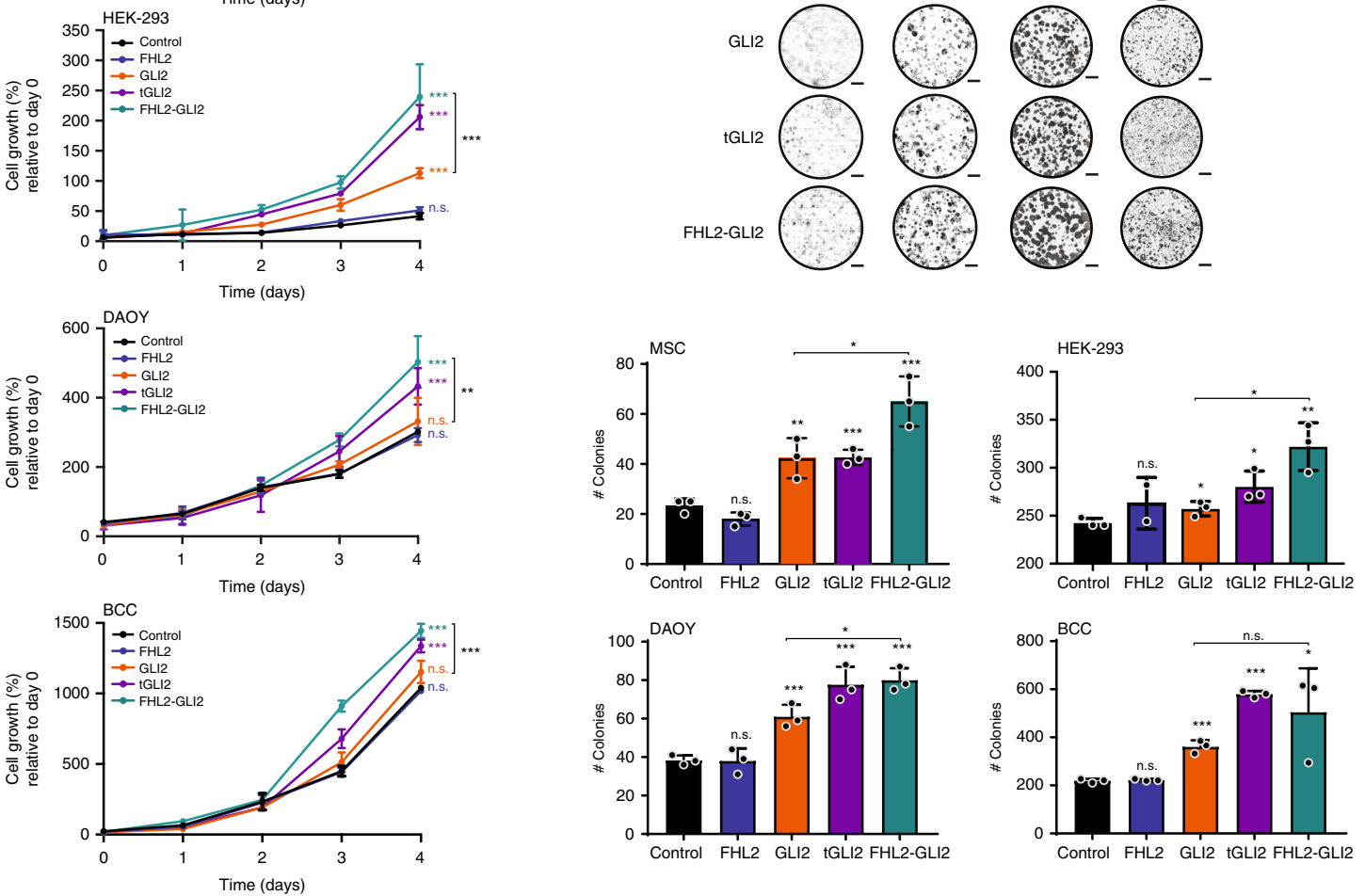

C
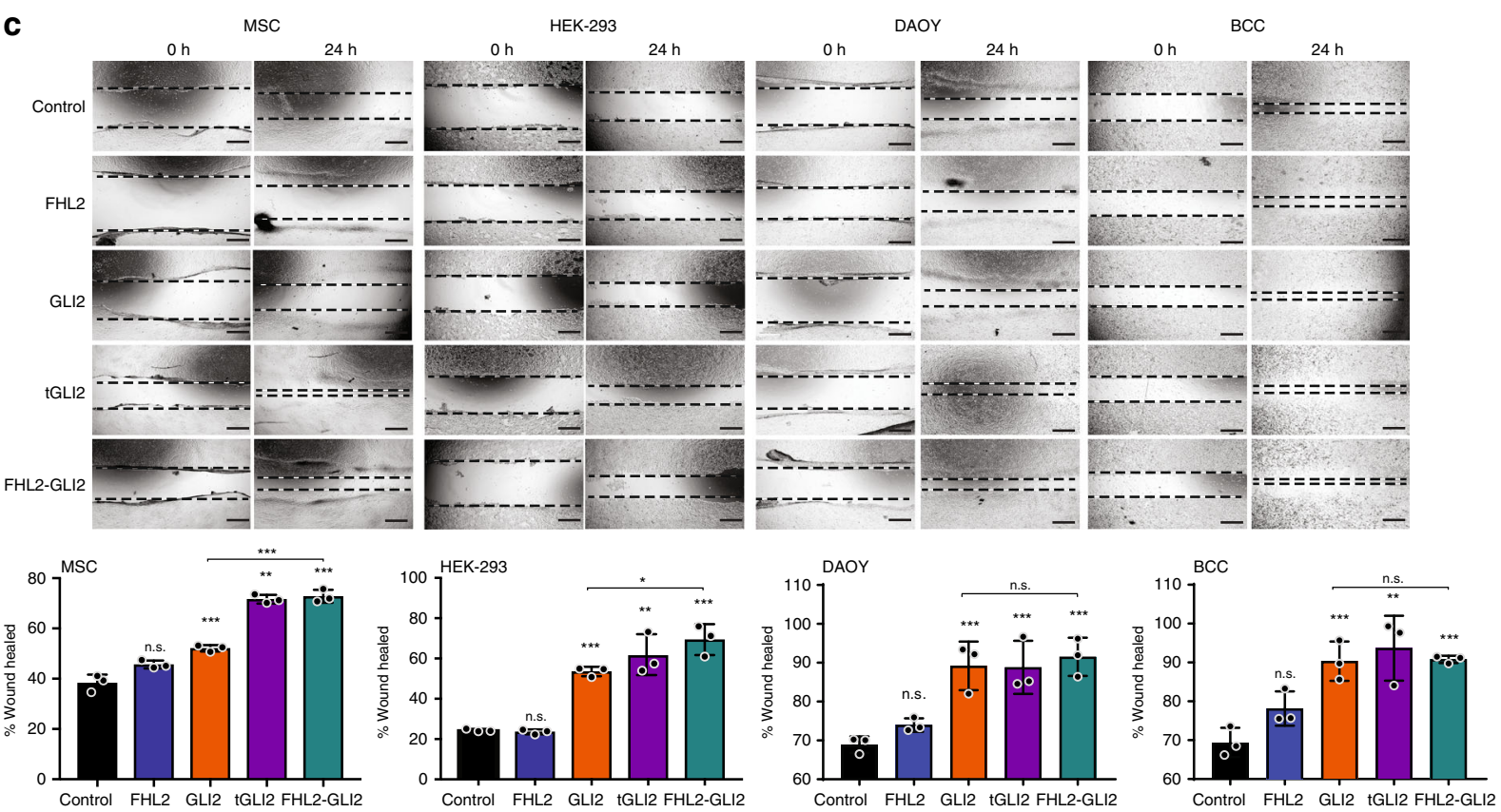

specific subtypes of neuronal tissue, but has also been found to be present in non-neoplastic human ovaries and sex cord-stromal tumors $^{31}$. Specifically, calretinin is expressed in theca interna, hilus and stromal cells ${ }^{31}$, and has proven a useful marker in the diagnosis of sex cord-stromal tumors ${ }^{32}$. Based on this understanding, we posited that cells expressing the fusion FHL2GLI2 would adopt an SST-like phenotype and express calretinin. We observed significantly higher levels of CALB2 transcripts in MSC and HEK-293 cells stably expressing tGLI2 or the FHL2-GLI2 fusion as compared to empty vector controls or cells 
Fig. 2 FHL2-GLI2 expression results in the acquisition of oncogenic properties in vitro. a Cell titer blue proliferation assay of immortalized mesenchymal stem cells (MSCs), HEK-293, medulloblastoma (DAOY), and human basal cell carcinoma (BCC) cells stably expressing empty vector (control), wild-type FHL2 (FHL2), wild-type GLI2 (GLI2), truncated GLI2 (tGLI2), or the FHL2-GLI2 fusion. b Representative images of colony formation assay of MSCs, HEK293, DAOY, and BCC cells stably expressing control, FHL2, GLI2, tGLI2, or FHL2-GLI2 (scale bars, 5 mm; top). Quantification of the number of colonies/ well compared to control (bottom). c Wound healing assay of MSCs, HEK-293, DAOY, and BCC cells stably expressing control, FHL2, GLI2, tGLI2 or FHL2GLI2. The migratory effects/wound area was assessed at 0 and $24 \mathrm{~h}$ (Scale bar, $500 \mu \mathrm{m}$; top) and quantified (bottom). In a-c, data are representative of at least three independent experiments. Error bars, s.d. of mean; n.s., not significant; ${ }^{\star} P<0.05,{ }^{\star \star} P<0.01$, ${ }^{\star \star \star} P<0.001$; two-tailed unpaired $t$-test.

expressing wild-type FHL2 or wild-type GLI2 (Fig. 3a). These findings were confirmed at the protein level; western blot and immunofluorescence analyses demonstrated increased levels of calretinin in MSC and HEK-293 cells expressing tGLI2 or the fusion gene FHL2-GLI2 than in controls (Fig. 3b, c). In addition, we tested FOXL2, a highly specific marker of ovarian sex cordstromal tumors ${ }^{10}$, and also found FOXL2 gene expression levels to be higher in cells transduced with tGLI2 and the FHL2-GLI2 fusion gene than in cells expressing empty vector controls, wildtype FHL2 or GLI2 alone (Fig. 3d), providing further evidence that the presence of the FHL2-GLI2 fusion gene results in the acquisition of some of the cardinal phenotypic characteristics of SSTs.

FHL2-GLI2 expression results in SHH pathway activation. GLI2 has been reported to play pivotal roles in the regulation of the SHH signaling pathway $22-24$. To define whether the FHL2GLI2 fusion gene would result in activation of this pathway in SSTs, we performed a differential gene expression analysis between the eight human SSTs subjected to RNA-sequencing, common-type high-grade serous ovarian carcinomas derived from TCGA ${ }^{11}(n=16)$ and other sex cord-stromal tumors $(n=$ 11). This analysis revealed an enrichment of $\mathrm{SHH}$ pathway genes, including GLI1, GLI2, HHIP, PTCH1, and PTCH2, in SSTs as compared to high-grade serous ovarian cancers and other sex cord-stromal tumors (Fig. 4a). To determine specifically whether SHH target genes, including PTCH1, PTCH2, HIF1A, HHIP, HHAT, GLI1/2/3, SMO, and SUFU, are co-expressed with the FHL2-GLI2 fusion gene in SSTs, we subjected SSTs $(n=11)$ and other ovarian sex cord-stromal tumors $(n=9$; thecomas, $n=3$; fibromas, $n=3$; GCTs $n=2$; and SLCT, $n=1)$ to NanoString gene expression analysis. The SHH GLI2 target genes tested were found to be co-expressed with the FHL2-GLI2 fusion gene. In addition, we observed that the $\mathrm{SHH}$ pathway genes were expressed also in SSTs with other GLI2 rearrangements, as well as in SSTs lacking GLI2 rearrangements based on the methods employed. Regardless of the presence and type of GLI2 rearrangements, SSTs were found to display significantly higher $\mathrm{SHH}$ pathway enrichment scores than other sex cord-stromal tumors $(p<0.001$, Wilcoxon rank test; Fig. 4b, Supplementary Fig. 7).

Given the evidence that the SHH pathway activation would potentially mediate the oncogenic properties of the FHL2-GLI2 fusion gene in human SSTs, we investigated the impact of the FHL2-GLI2 fusion expression on this pathway in our in vitro models. Consistent with the observations derived from the analysis of human SSTs, stable expression of tGLI2 and the FHL2-GLI2 fusion in MSC, HEK-293, DAOY, and BCC cells resulted in a significant increase in the expression levels of the $\mathrm{SHH}$ receptor $\mathrm{PTCH} 1$ and/or GLI1/CCND1 as compared to empty vector, wild-type FHL2 and wild-type GLI2 (Fig. 4c). For BCCs, given the very low endogenous levels of GLI1 gene/protein expression, CCND1 rather than GLI1 was assessed. Again, increased PTCH1 and GLI1/CCND1 gene expression was also observed upon wild-type GLI2 expression, however at levels significantly lower than in tGLI2 and FHL2-GLI2 cells, both of which harbor GLI2 lacking the repression domain (Fig. 4c). This finding was further supported by quantitative western blot analyses, which demonstrated increased levels of PTCH1 and GLI1 or CCND1 proteins upon tGLI2 and FHL2-GLI2 expression in all four cell models, although the increases in PTCH1 were not significant in the DAOY and BCC cells (Fig. 4d).

These findings in human SSTs and in vitro models provide evidence to suggest that the oncogenic behavior of SSTs can at least in part be explained by activation of the $\mathrm{SHH}$ pathway, and the potential of GLI2 rearrangements to serve as a marker for these uncommon ovarian neoplasms.

FHL2-GLI2 activates the SHH pathway via GLI2 target promoters. Given the role of the $\mathrm{SHH}$ pathway in the oncogenesis of SSTs, we sought to define the mechanistic basis of the activation of the $\mathrm{SHH}$ pathway in these tumors. GLI2, a key member of the SHH pathway, has a nuclear localization where it acts as a transcription factor ${ }^{33}$. Immunofluorescence utilizing an anti-FLAG antibody revealed that akin to wild-type GLI2, which displayed a nuclear localization in HEK-293 cells expressing the wild-type GLI2 vector, tGLI2 and the FHL2-GLI2 fusion proteins were expressed in the nuclear compartment (Fig. 5a). These findings demonstrate that deletion of the $\mathrm{N}$-terminal domain of GLI2 does not abrogate its nuclear localization.

To test whether tGLI2 and the FHL2-GLI2 fusion proteins would activate GLI-responsive (RE) elements akin to wild-type GLI2, we performed a luciferase reporter assay with a specific GLI-RE containing GLI DNA binding sites in HEK-293, DAOY and BCC cells stably expressing empty vector, wild-type FHL2, wild-type GLI2, tGLI2, and FHL2-GLI2 (Fig. 5b, Supplementary Fig. 8). Increased activation of GLI-RE was seen in cells expressing wildtype GLI2, tGLI2, and the FHL2-GLI2 fusion but GLI-RE activation was most enhanced in tGLI2 or FHL2-GLI2 fusion gene expressing cells (Fig. 5b). Furthermore, we observed that mutation of the R338A-K339A amino acids in the Zinc Finger 5 of GLI2, which are highly conserved between all GLI family members and reported to be required for DNA binding ${ }^{33}$, resulted in inhibition of the capacity of the FHL2-GLI2 fusion protein to activate the SHH pathway (Fig. 5b). These data provide additional evidence that DNA binding of the FHL2-GLI2 fusion protein is necessary for the downstream activation of the $\mathrm{SHH}$ pathway.

We next investigated the ability of the FHL2-GLI2 fusion protein, which lacks the $\mathrm{N}$-terminal binding domain, to bind to the Suppressor of fused homolog (SUFU) protein. SUFU is a known negative regulator of the $\mathrm{SHH}$ signaling pathway, which inhibits GLI2 through direct binding to specific regions located in the $\mathrm{N}$-terminal and $\mathrm{C}$-terminal regions ${ }^{34}$. Immunoprecipitation of SUFU revealed that both the FHL2-GLI2 fusion and tGLI2 proteins do not bind or only weakly bind to SUFU as compared to the wild-type GLI2 protein in HEK-293 cells (Fig. 5c). Luciferase experiments confirmed that SUFU overexpression is able to suppress SHH activity in HEK-293 cells expressing tGLI2 and the FHL2-GLI2 fusion protein (Fig. 5c). Inhibition of SHH activation in cells expressing tGLI2 or the FHL2-GLI2 fusion protein, which lack the N-terminal region of GLI2, likely stems from the binding of SUFU to the retained C-terminal domain of GLI2 ${ }^{34}$. These findings were further supported by treatment of 
a
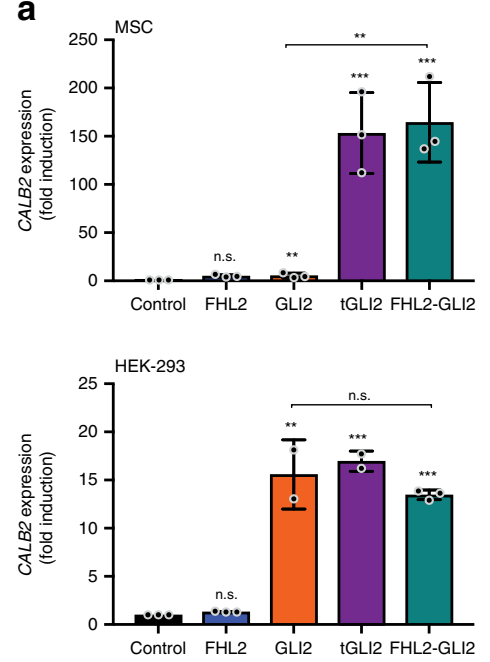

C
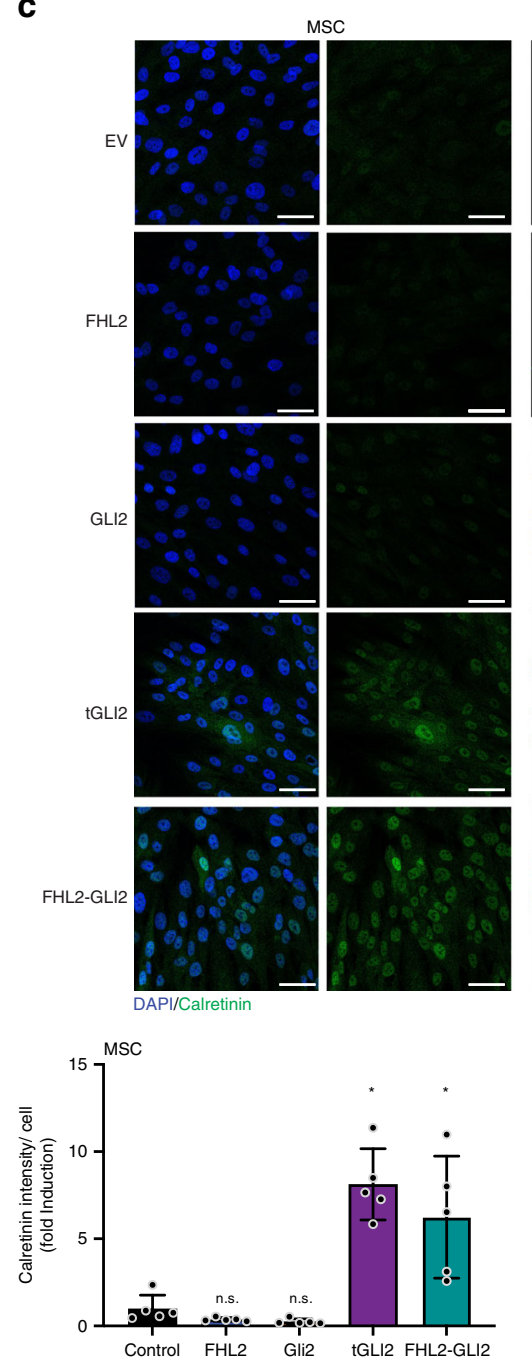

b
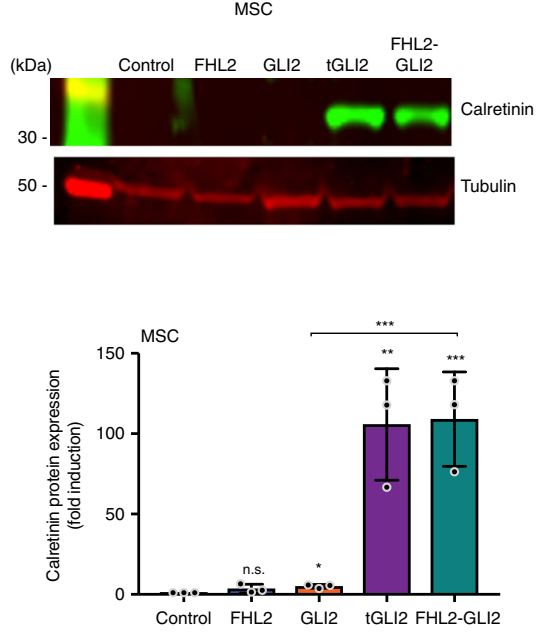

d
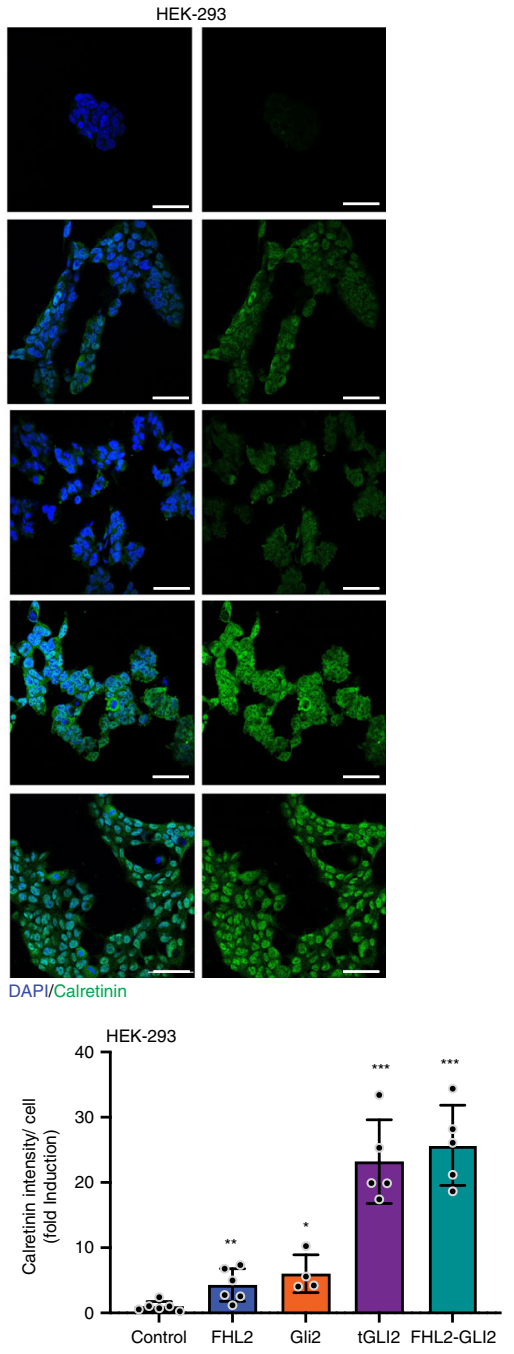
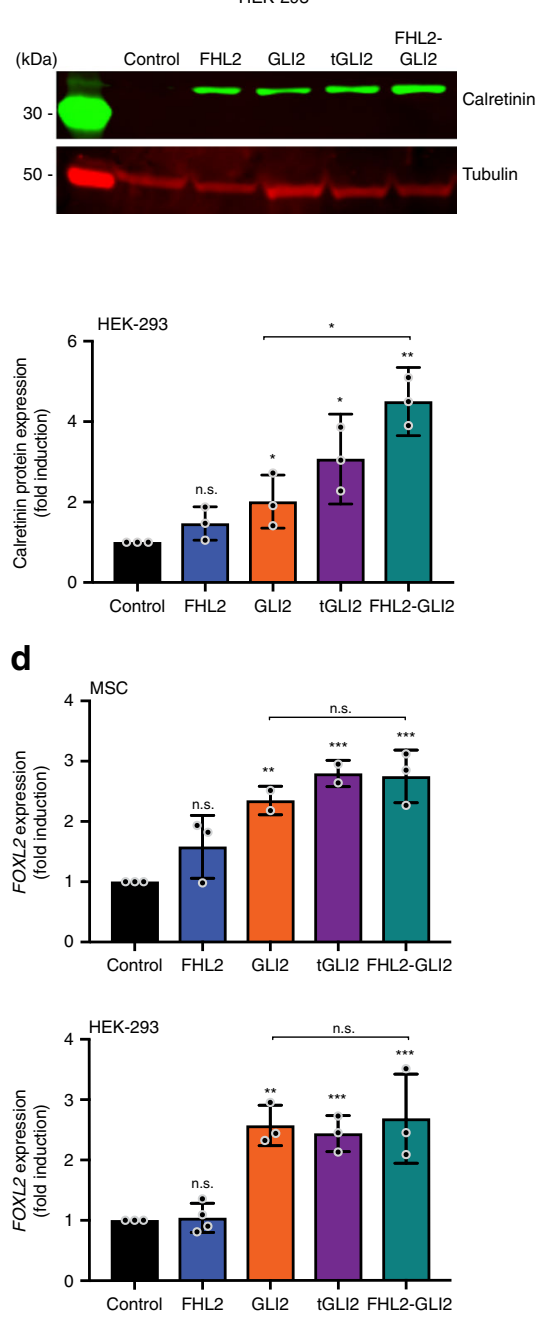

HEK-293, DAOY, and BCC cells with GANT61, a small molecule inhibitor that specifically targets the GLI1/2 DNA binding site, which resulted in decreased proliferation in all cells, but predominantly in DAOY and BCC cells expressing tGLI2 or FHL2-GLI2 (Fig. 5d). HEK-293 cells were found to be markedly sensitive to GANT61 treatment and proliferation was decreased in all vectors and conditions tested.
Finally, we sought to define whether the FHL2-GLI2 fusion protein binds to GLI target genes. We performed chromatin immunoprecipitation (ChIP) with FLAG antibody, which demonstrated the recruitment of the FHL2-GLI2 fusion protein on two different regions of both the GLI1 and PTCH1 promoters (promoters 1 and 2) in MSC and HEK-293 cells expressing this

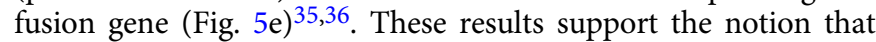


Fig. 3 FHL2-GLI2 expression results in the expression of sex cord markers in vitro. a Quantitative assessment of CALB2 transcripts in immortalized mesenchymal stem cells (MSCs) and HEK-293 cells stably expressing empty vector (control), wild-type FHL2 (FHL2), wild-type GLI2 (GLI2), truncated GLI2 (tGLI2), or the FHL2-GLI2 fusion. Expression levels were normalized to GAPDH expression, and comparisons of mRNA expression levels were performed relative to control. b Representative western blot analysis of calretinin protein levels in MSCs and HEK-293 cells stably expressing control, FHL2, GLI2, tGLI2, or FHL2-GLI2. Tubulin was used as protein loading control. Quantification (bottom) of protein levels as compared to control. c Representative confocal micrographs of immunofluorescence analysis of calretinin (green) and 4-6-diamidino-2-phenylindole (DAPI, blue) in MSCs and HEK-293 cells stably expressing control, FHL2, GLI2, tGLI2, or FHL2-GLI2 (scale bars, $50 \mu \mathrm{m}$ ). Quantification (bottom) of calretinin intensity/cell relative to control. d Quantitative assessment of FOXL2 transcripts in MSCs and HEK-293 cells stably expressing control, FHL2, GLI2, tGLI2, or FHL2-GLI2. Expression levels were normalized to GAPDH expression, and comparisons of mRNA expression levels were performed relative to control. In a-d, data are representative of at least three independent experiments. Error bars, s.d. of mean; n.s., not significant; ${ }^{\star} P<0.05,{ }^{\star \star} P<0.01$, ${ }^{\star \star \star} P<0.001$; two-tailed unpaired $t$-test.

the fusion protein binds directly to promoters of genes with GLI binding sites, thereby leading to activation of the $\mathrm{SHH}$ signaling pathway.

Oncogenic properties reversed by $\mathrm{SHH}$ pathway inhibition. Antagonists targeting downstream effectors of the SHH pathway, specifically smoothened (SMO) and GLI1, have been developed as cancer therapeutics and are FDA-approved in the treatment of basal cell carcinomas ${ }^{37}$, tumors driven by the activation of the $\mathrm{SHH}$ pathway. Given the activation of the $\mathrm{SHH}$ pathway in human SSTs and upon FHL2-GLI2 expression in our in vitro models, we assessed whether inhibition of the $\mathrm{SHH}$ pathway would result in reversal of the oncogenic phenotype induced by FHL2-GLI2 expression. Upon treatment of MSC and/or HEK293, DAOY, and BCC cells stably expressing empty vector, wildtype FHL2, wild-type GLI2, tGLI2, or the FHL2-GLI2 fusion protein with Vismodegib (Fig. 6a-c) and cyclopamine (Supplementary Fig. 9a-c), both highly specific inhibitors of $\mathrm{SMO}^{37}$, we observed a significant effect on proliferation and colony formation in cells expressing tGLI2 and the FHL2-GLI2 fusion protein (Fig. 6a, b, Supplementary Fig. 9a, b). We also observed a significant decrease in migration of MSC and/or HEK-293, DAOY, and BCC cells expressing tGLI2 and the FHL2-GLI2 fusion protein as compared to controls when treated with Vismodegib (Fig. 6c) or cyclopamine (Supplementary Fig. 9c).

Taken together, these results implicate the $\mathrm{SHH}$ pathway in the phenotype observed in cells stably transduced with the fusion FHL2-GLI2 and provide evidence for the underlying biological basis and pathogenesis of SSTs of the ovary.

\section{Discussion}

Here, we demonstrate that SSTs of the ovary harbor recurrent GLI2 rearrangements, with FHL2 being the most frequent partner gene. We further show through an analysis of other ovarian sex cord-stromal tumors and 33 different cancer types that this FHL2-GLI2 fusion is likely pathognomonic for SSTs, providing a genotypic-phenotypic correlation in ovarian neoplasms.

GLI-transcription factors have been shown to be mediators of SHH signaling, and constitutive activation of the SHH/GLI signaling cascade has been implicated in numerous human malignancies, including skin, lung and prostate ${ }^{22,38}$. N-terminally truncated Gli2, unlike the full-length protein, has been reported to activate Shh target genes in transgenic mouse embryos ${ }^{39}$, suggesting that the modulation of the $\mathrm{N}$-terminal repression domain is a mechanism of Shh signaling activation. The $\mathrm{SHH}$ pathway has been shown to play a prominent role in the communication between granulosa cells and developing theca cells $^{40,41}$, and FHL2 is ubiquitously expressed in benign ovarian tissue and ovarian granulosa cells ${ }^{19}$. The FHL2-GLI2 fusion gene identified here likely has an oncogenic mechanism consistent with that of several other fusion genes, where the expression of the $3^{\prime}$ partner gene is driven by the regulatory elements of the ubiquitously expressed $5^{\prime}$ partner gene ${ }^{42}$. In addition, the exons of GLI2 included in the FHL2-GLI2 fusion gene lack the GLI2 repression domain but maintain its DNA binding domain. Hence, we propose a model where the FHL2-GLI2 fusion gene results in the overexpression of a truncated GLI2 (lacking its repression domain), given that its expression is under the FHL2 promoter, a protein ubiquitously expressed in ovarian stromal cells (i.e., the likeliest cell of origin of SSTs). Consistent with this model, the FHL2-GLI2 fusion and tGLI2 lacking the repression domain display nuclear localization (Fig. 5a) and result in activation of the SHH signaling pathway (Fig. 5b). In addition, the FHL2-GLI2 fusion protein was found to bind to the promoters of the GLI2 target genes PTCH1 and GLI1 (Fig. 5e), and was observed to require an intact GLI2 DNA binding site for the activation of the $\mathrm{SHH}$ pathway (Fig. 5b). Furthermore, the oncogenic effects of FHL2-GLI2 expression observed in cells could be reversed through pharmacological inhibition of the $\mathrm{SHH}$ pathway using three chemical inhibitors, further demonstrating the role of the GLI2-mediated SHH signaling pathway activation and dependency in cells expressing FHL2-GLI2.

We have demonstrated here that SSTs of the ovary are driven by GLI2 activation through the expression of GLI2-rearrangements. Whilst other rare types of ovarian neoplasms have been found to be underpinned by highly recurrent mutations, such as FOXL2 hotspot mutations in adult-type granulosa cell tumors ${ }^{9}$ or SMARCA4 loss-of-function mutations in small cell carcinomas of the ovary hypercalcemic type ${ }^{12}$, SSTs were found to be characterized by recurrent and likely pathognomonic FHL2-GLI2 fusion genes. Other rare types of tumors with a relatively favorable clinical behavior have also been shown by our group and others to be driven by activating mutations and/or fusion genes. For example, adenoid cystic carcinomas of the breast are primarily driven by the MYB-NFIB fusion gene in $\sim 80 \%$ of cases $^{43,44}$; in breast adenoid cystic carcinomas lacking these fusions, MYBL1 rearrangements and $M Y B$ gene amplification have been reported as alternative mechanisms resulting in MYB overexpression and activation of MYB targets ${ }^{45}$. Likewise, $>70 \%$ of polymorphous adenocarcinomas of the salivary glands are characterized by pathognomonic PRKD1 hotspot mutations; 46 those with wild-type PRKD1 have been shown to harbor PRKD1/ $2 / 3$ rearrangements ${ }^{47}$. Consistent with these observations, in this study, we identified a DYNLL1-GLI2 fusion gene in an SST lacking the FHL2-GLI2 fusion gene. Further studies are warranted to define the fusion partners of the SSTs with GLI2 rearrangements but lacking FHL2-GLI2 and DYNLL1-GLI2 fusions, and to determine the mechanisms leading to GLI2 and SHH signaling pathway activation in SSTs lacking fusion genes involving GLI2.

This study has several limitations. Due to the retrospective multi-institutional nature of our study, follow-up information was unavailable, and the impact of the FHL2-GLI2 fusion on patient outcome could not be assessed. We did not identify a GLI2 rearrangement or alternative driver genetic alteration in $19 \%(5 / 26)$ of the SSTs studied. In addition, in three SSTs, FISH 
a

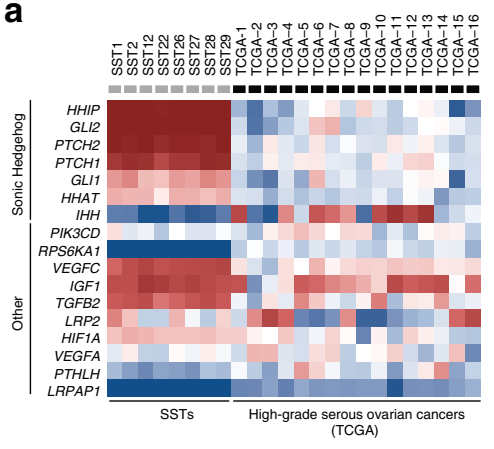

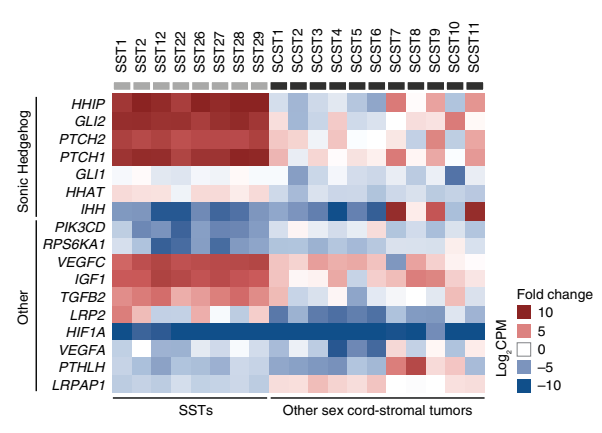

C
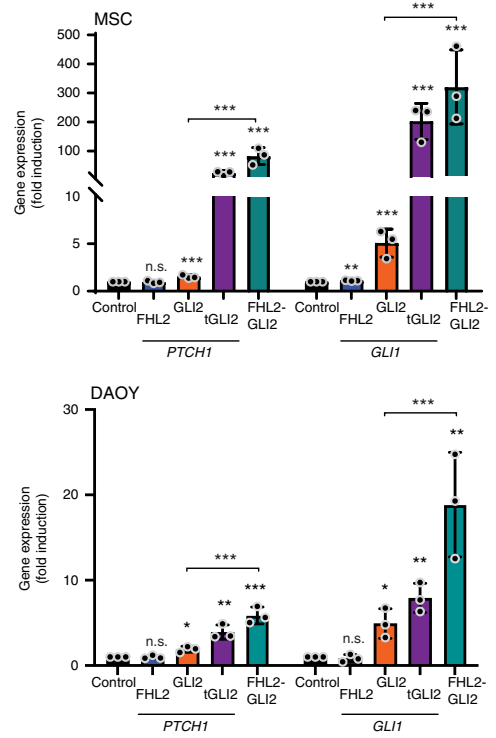
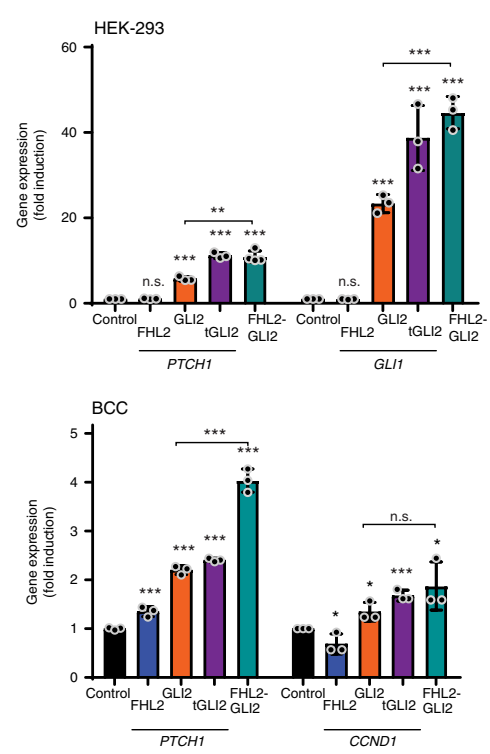

b

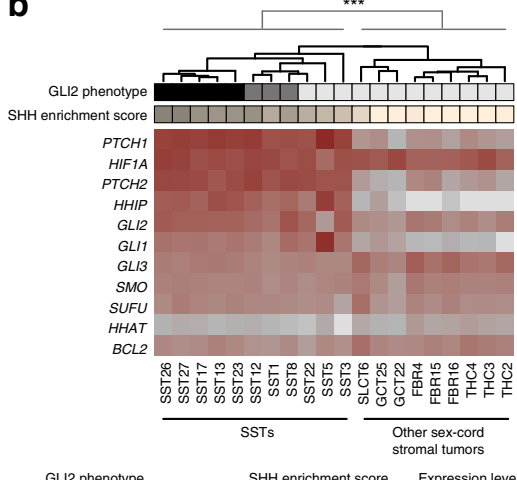

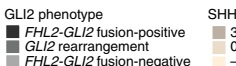

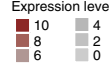
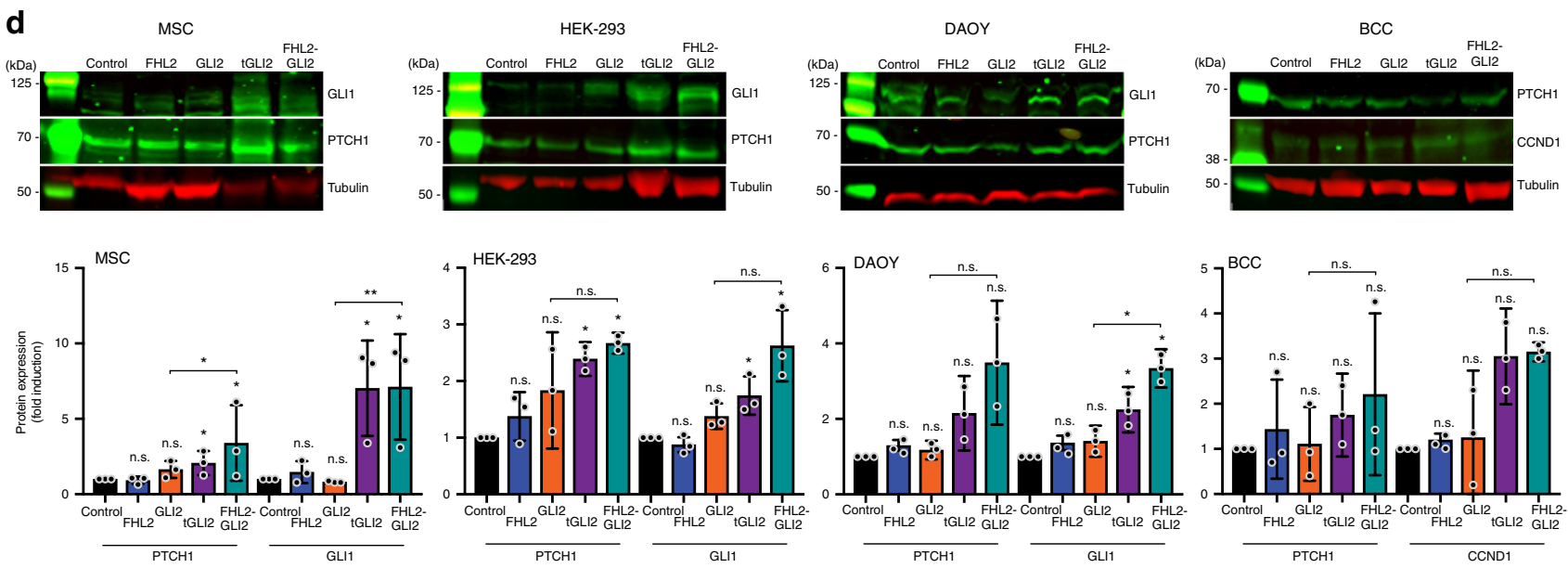

Fig. 4 Expression of the FHL2-GLI2 fusion results in the activation of the Sonic Hedgehog pathway. a Differential gene expression analysis of human sclerosing stromal tumors (SSTs) subjected to RNA-sequencing ( $n=8$, this study) and high-grade serous ovarian carcinomas ( $n=16$; The Cancer Genome Atlas) and other sex cord-stromal tumors (SCSTs, $n=11$; this study). Gene expression fold-change is color-coded according to the legend. Only genes significantly differentially expressed ( $P<0.05$; two-tailed unpaired $t$-test) are shown. CPM, count per million. $\mathbf{b}$ Expression levels of Sonic Hedgehog (SHH) pathway genes in human SSTs $(n=11)$ and other sex-cord stromal tumors $(n=9)$ as defined using NanoString. Expression levels and SHH enrichment scores are color-coded according to the legends. ${ }^{\star \star \star} P<0.001$, Wilcoxon rank test. Hierarchical clustering was performed using complete linkage and Euclidian distance. c Quantitative assessment of the Sonic Hedgehog pathway PTCH1 and GLI1 transcripts in immortalized mesenchymal stem cells (MSCs), HEK-293 and medulloblastoma (DAOY) cells and of PTCH1 and CCND1 transcripts in human basal cell carcinoma (BCC) cells stably expressing empty vector (control), wild-type FHL2 (FHL2), wild-type GLI2 (GLI2), truncated GLI2 (tGLI2), or FHL2-GLI2. Expression levels were normalized to GAPDH expression, and comparisons of mRNA expression levels were performed relative to control. d Representative western blot analysis of PTCH1 and GLI1 protein expression in MSC, HEK-293 and DAOY cells and of PTCH1 and CCND1 in BCC cells stably expressing control, FHL2, GLI2, tGLI2, or FHL2-GLI2. Tubulin was used as protein loading control. Quantification (below) of protein levels as compared to control. In c-d, data are representative of at least three independent experiments. Error bars, s.d. of mean; n.s., not significant; ${ }^{\star} P<0.05,{ }^{\star \star} P<0.01,{ }^{\star \star \star} P<0.001$; two-tailed unpaired $t$-test. 
a
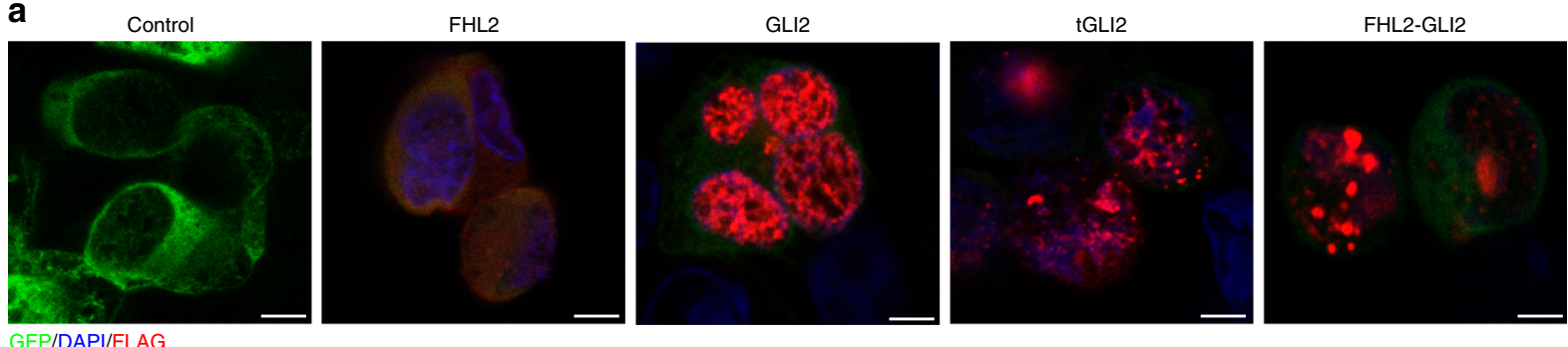

b
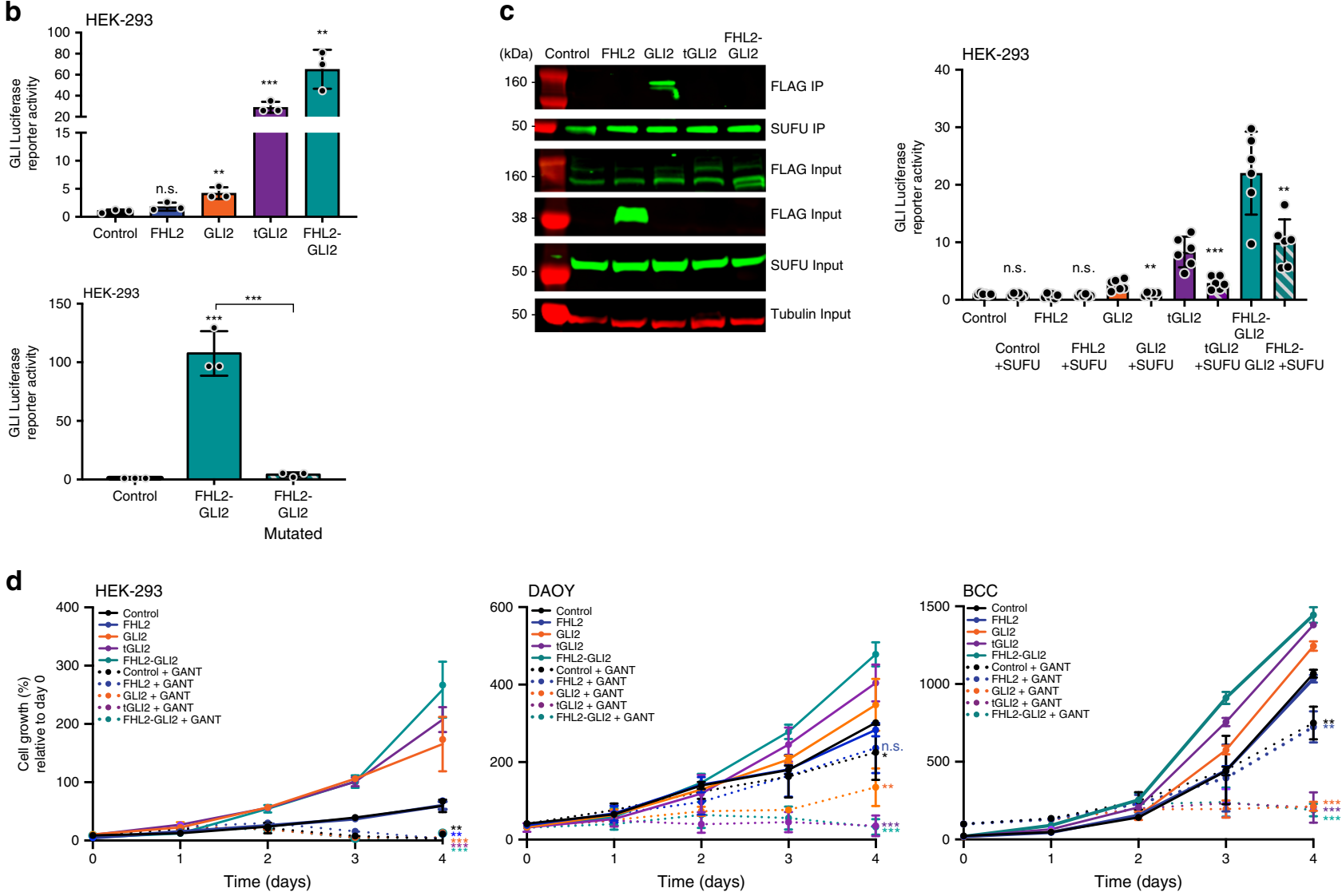

e
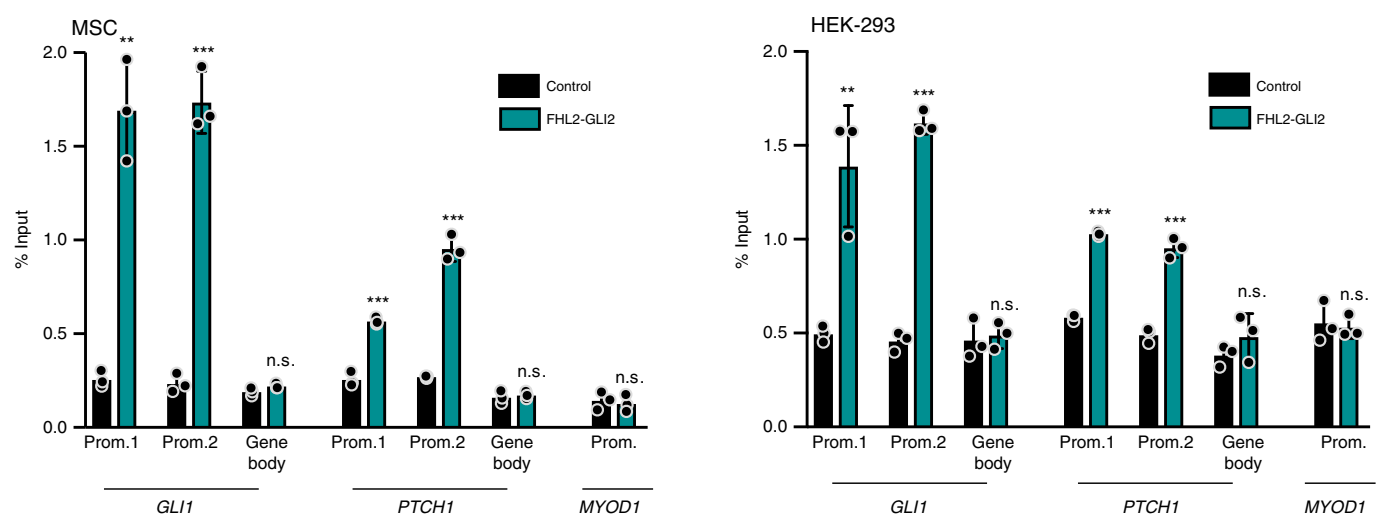

revealed the presence of GLI2 rearrangements, however the partner genes could not be identified. Of note, GLI2 protein expression and $\mathrm{SHH}$ pathway activation were also observed in SSTs lacking any of the GLI2 rearrangements identified here (Fig. 4, Supplementary Figs. 2 and 6), providing evidence to suggest that mechanisms other than the FHL2-GLI2 and DYNLL1-GLI2 fusion genes may lead to the constitutive activation of GLI2 in these lesions. Further studies, ideally utilizing frozen samples of SSTs with GLI2 rearrangements other than those described in this study or lacking GLI2 rearrangements altogether are warranted to define their driver alterations. Finally, due to the lack of representative cell line models derived from human SSTs, we established cell models using MSC, HEK-293, DAOY, and BCC cells with stable expression of the FHL2-GLI2 fusion and tGLI2. Despite HEK-293 cells not being representative of the likely cell of origin, FHL2-GLI2 expression resulted in the 
Fig. 5 FHL2-GLI2 activates the Sonic Hedgehog pathway through DNA binding in GLI target promoters. a Representative confocal micrographs of immunofluorescence analysis of FLAG (red), 4-6-diamidino-2-phenylindole (DAPI, blue), and GFP (green) in HEK-293 cells stably expressing empty vector (control), wild-type FHL2 (FHL2), wild-type GLI2 (GLI2), truncated GLI2 (tGLI2), or FHL2-GLI2. Scale bars, $10 \mu \mathrm{m}$. b GLI response element (GLI-RE) luciferase reporter assay of HEK-293 cells stably expressing control, FHL2, GLI2, tGLI2, or FHL2-GLI2 (top), and GLI-RE promoter activity in HEK-293 transiently transfected with control, FHL2-GLI2 and FHL2-GLI2 with R338A-K339A mutations in the Zinc Finger 5 of GLI2 required for DNA binding (FHL2GLI2 Mutated; bottom). SV40-Renilla was used to normalize transfection efficiency. c Immunoprecipitation assay with SUFU antibody of HEK-293 cells stably expressing control, FHL2, GLI2, tGLI2, or FHL2-GLI2. Western blot analysis using anti-FLAG and anti-SUFU antibodies, and tubulin as loading control (left). GLI-RE promoter activity in HEK-293 stably expressing control, FHL2, GLI2, tGLI2, or FHL2-GLI2 transfected with SUFU or control (right). d Cell titer blue proliferation assay of HEK-293, DAOY and BCC cells stably expressing control, FHL2, GLI2, tGLI2, or FHL2-GLI2 treated with $20 \mu \mathrm{M}$ GANT61 or vehicle control (DMSO). GANT, GANT61. e FLAG Chromatin Immunoprecipitation (ChIP) assay of GLI1 and PTCH1 promoters (promoter 1 and 2) in MSC and HEK-293 cells stably expressing either control or FHL2-GLI2. GLI1 and PTCH1 gene body and MYOD1, gene promoters not under GLI regulation, were used as negative controls. In $\mathbf{b}-\mathbf{d}$, data are representative of at least three independent experiments. Error bars, s.d. of mean; n.s., not significant; ${ }^{\star} P<0.05$, ${ }^{\star \star} P<0.01,{ }^{\star \star \star} P<0.001 ;$ two-tailed unpaired $t$-test.

acquisition of phenotypic characteristics of sex cord-stromal tumors, including expression of sex cord markers and $\mathrm{SHH}$ pathway activation. Whilst we utilized DAOY and BCC cell lines given their dependence on the $\mathrm{SHH}$ pathway ${ }^{22,48,49}$, as expected in the setting of preexisting activation of GLI-signaling, the effects of FHL2-GLI2 expression in DAOY and BCC cell lines, albeit mostly significant, were not as overt as those observed in MSCs and HEK-293 cells.

Despite these limitations, we report on the pathognomonic fusion gene FHL2-GLI2 in SSTs of the ovary, which results in the activation of the $\mathrm{SHH}$ pathway. Our findings provide evidence to suggest that GLI2 rearrangements are likely drivers of SSTs and may serve as potential ancillary markers for the diagnosis of these tumors, and that GLI2 should be included in panels surveying fusion genes in human cancers. Finally, our results on the $\mathrm{SHH}$ pathway activation in SSTs provide a potential therapeutic avenue for patients with SSTs, with FDA-approved SHH pathway inhibitors being available.

\begin{abstract}
Methods
Subjects and samples. Following approval by the Institutional Review Boards (IRBs)/local ethics committees of the authors' institutions, unstained tissue sections from formalin-fixed paraffin-embedded (FFPE) SSTs were retrieved from Fudan University Cancer Center's consultation cases (Shanghai, China) and Massachusetts General Hospital (MGH, MA, USA). Patient consents were obtained following the respective IRB protocols approved by the authors' institutions, and samples were anonymized before analysis. All tumors were centrally reviewed by four pathologists (R.B., J.S.R.-F., R.A.S. and E.O.), and 26 tumors were classified as SSTs and included in this study.

The discovery and validation series comprised 8 and 18 SSTs, respectively (Supplementary Table 1, Supplementary Fig. 1). The 8 SSTs from the discovery series were subjected to microdissection, RNA extraction and RNA-sequencing, and to microdissection of the tumor and normal tissues, DNA extraction and whole-exome $(n=3)$ or MSK-IMPACT $(n=5)$ sequencing (Fig. 1, Supplementary Table 1, Supplementary Fig. 1). FFPE-derived RNA from all microdissected SSTs were subjected to RT-PCR ( $n=26$; discovery and validation series), and 13 SSTs were subjected to fluorescence in situ hybridization (FISH; Fig. 1, Supplementary Fig. 1, Supplementary Table 4). In addition, RNA from other ovarian sex cordstromal tumors was also subjected to RT-PCR $(n=48)$ and/or FISH $(n=9$; Figs. 1, 4, Supplementary Fig. 1, Supplementary Table 4).
\end{abstract}

Whole-exome and targeted massively parallel sequencing. Microdissected tumor and normal DNA from 3 SSTs was subjected to whole-exome sequencing (WES), and microdissected tumor DNA from 5 SSTs to MSK-IMPACT sequencing (Supplementary Fig. 1, Supplementary Table 1), a massively parallel sequencing assay targeting all exons and selected introns of 410 cancer-related genes ${ }^{16}$. Sequencing was performed at Memorial Sloan Kettering Cancer Center's (MSKCC's) Integrated Genomics Operation (IGO) using validated protocols ${ }^{50,51}$. Sequencing data were analyzed and mutations identified using validated bioinformatics methods ${ }^{50,51}$. Mutations affecting hotspot codons were annotated according to Chang et al. ${ }^{52}$. Copy number alterations (CNAs) and loss of heterozygosity (LOH) were defined using FACETS ${ }^{53}$ as described ${ }^{50,51}$. ABSOLUTE $(\mathrm{v} 1.0 .6)^{54}$ was employed to determine the cancer cell fraction (CCF) of each mutation ${ }^{50,51}$.
RNA-sequencing and fusion gene identification. Eight SSTs were subjected to paired-end RNA-sequencing (Supplementary Fig. 1; Supplementary Table 1) using validated protocols at MSKCC's IGO 51,55 . Read pairs supporting fusion transcripts were identified using INTEGRATE ${ }^{56}$, deFuse ${ }^{57}$ and FusionCatcher ${ }^{58}$, as described ${ }^{51,55}$. To filter out common alignment artifacts and normal transcriptional variation, we removed fusion gene and read-through candidates that were also found in a set of 297 normal samples from The Cancer Genome Atlas (TCGA) ${ }^{59}$ The remaining in-frame candidate fusion genes were annotated to predict their oncogenic/driver potential using OncoFuse (v1.0.9b2) ${ }^{17}$. The presence of identified fusion gene candidates was assessed in other cancer types using the Tumor Fusion Gene Data Portal ${ }^{28}$, which comprises 20,731 fusion genes across 33 cancer types $(n=9950$; October 2019).

Differential gene expression analysis. For differential gene expression analysis, RNA-sequencing data of 8 SSTs (discovery cohort), of 11 other sex cord-stromal tumors (this study; adult-type granulosa cell tumors, $n=6$; juvenile-type granulosa cell tumors, $n=2$; and Sertoli-Leydig cell tumors, $n=3$ ) and of high-grade serous ovarian carcinomas derived from TCGA ${ }^{11}(n=16$; GDC Legacy Archive, https:// portal.gdc.cancer.gov/legacy-archive/) were employed. Transcriptomic analysis was performed in the R environment using edgeR (v3.24.3) ${ }^{60}$. Genes with less than 0.2 count per million (CPM) were labeled as poorly expressed across all libraries and removed from further analyses. Raw counts were normalized using an upper quartile of M-values approach ${ }^{61}$, and dispersion estimates over all genes were carried out with the quantile-adjusted conditional maximum likelihood method ${ }^{62}$. Resulting $p$-values were corrected for multiple testing using the Benjamini-Hochberg false discovery rate $^{63}$. Furthermore, Reads Per Kilobase Million (RPKM) values were estimated from raw counts normalizing for sequencing depth and adjusting for length of genes.

FHL2-GLI2 fluorescence in situ hybridization. FHL2-GLI2 fusion gene assessment by FISH was performed using validated protocols at MSKCC's Molecular Cytogenetics Core ${ }^{64}$. Probes for detection of the FHL2-GLI2 fusion gene consisted of a three-color probe mix, composed of bacterial artificial chromosomes (BACs) mapping to 5' GLI2 (orange), 3' GLI2 (red), and 5' FHL2 (green). To screen for the presence of GLI2 rearrangements in SSTs lacking FHL2-GLI2/DYNLL1-GLI2 fusions by RNA-sequencing and/or RT-PCR, probes for the detection of the GLI2 gene consisted of a dual-color probe mix, composed of the same BACs as above mapping to $5^{\prime}$ GLI2 (yellow) and 3' GLI2 (green). At least 10 images per tumor region were captured and at least 50 non-overlapping interphase nuclei with welldelineated contours were analyzed for the presence of the FHL2-GLI2 fusion or GLI2 rearrangements. SSTs were considered positive for the FHL2-GLI2 fusion gene or for GLI2 rearrangements if $>15 \%$ of the cells displayed at least one $5^{\prime} \mathrm{FHL2}-$ 3'GLI2 fusion or 5'GLI2 or 3'GLI2 signal.

NanoString gene expression analysis. A NanoString nCounter gene expression assay using a custom CodeSet panel of SSH pathway genes was performed according to the manufacturer's instructions (NanoString Technologies). NanoString nCounter data were analyzed using the R Bioconductor package NanoStringDiff ${ }^{65}$ an approach which facilitates analysis of nCounter data using a negative binomial generalized linear model (GLM) with differential expression analysis performed using model-based linear contrasts, and associated inference based on likelihood ratio tests. The negative binomial GLM incorporates normalization factors for positive controls, background noise and the 5 housekeeping genes. Single sample Gene Set Enrichment (ssGSEA) analysis ${ }^{66}$ was used to define the Sonic Hedgehog (SHH) enrichment score for each sample. Hierarchical cluster analysis of gene expression data was performed using complete linkage and Euclidian distance.

Single-molecule RNA in situ hybridization. RNA in situ hybridization (RNA-ISH) experiments were performed using RNAscope, according to the manufacturer's instructions ${ }^{67}$. Paired double- $Z$ oligonucleotide probes were designed against target RNA: BA-Hs-GLI2-3EJ (Cat no. 801211, NM_005270.4, 3zz pair, nt 850-1221), 
a
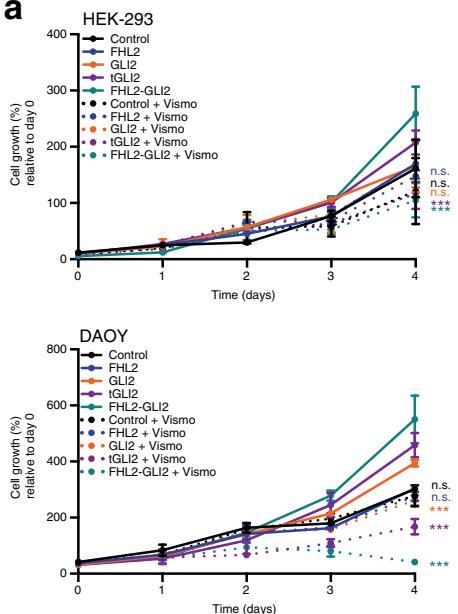

b

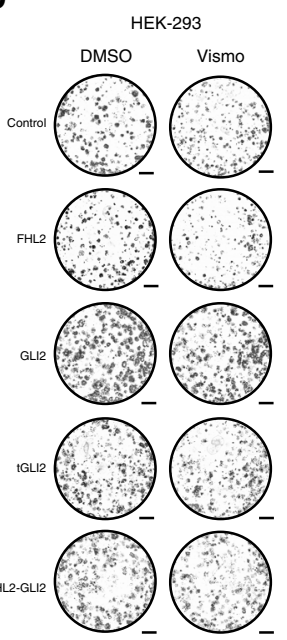

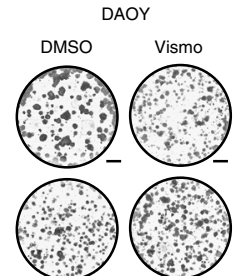
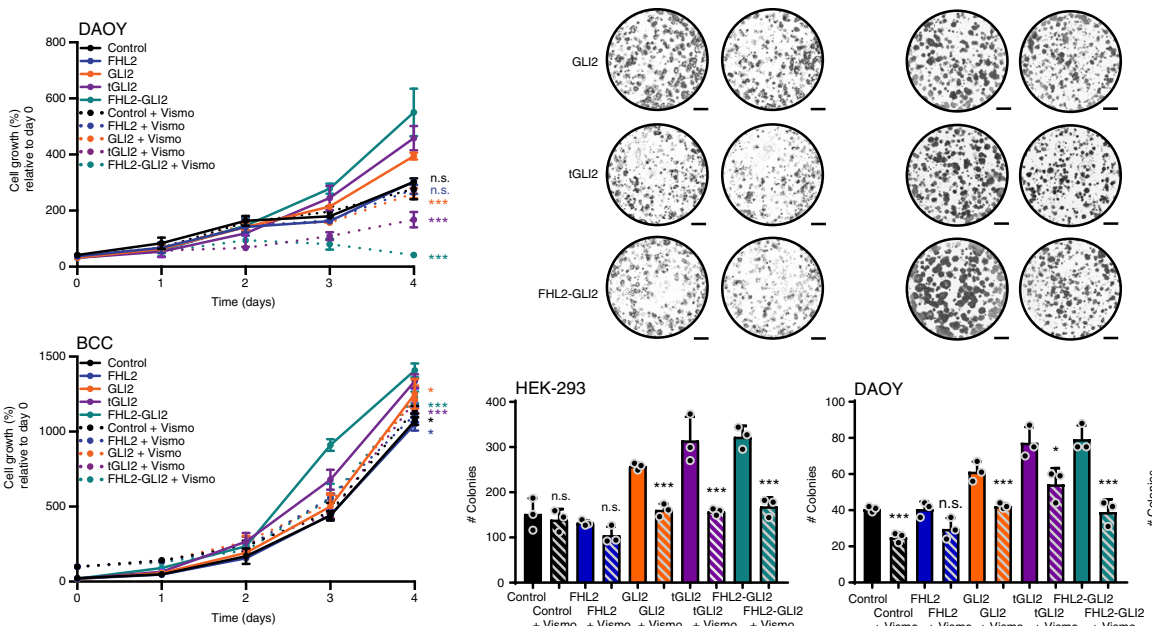

DAOY

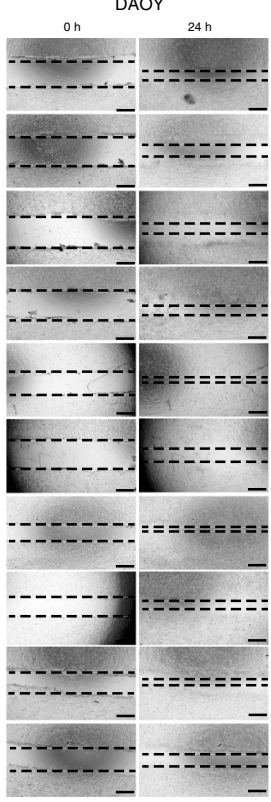

C
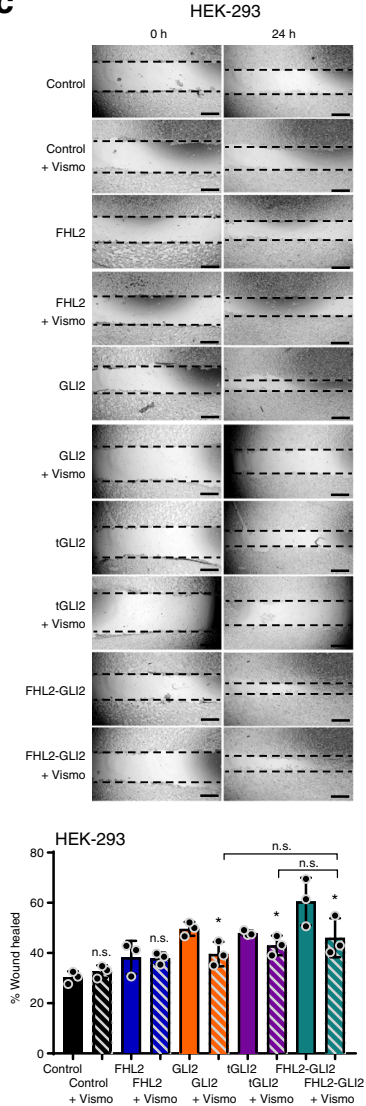

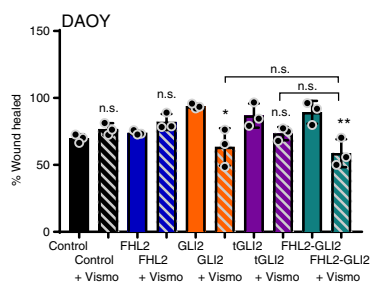

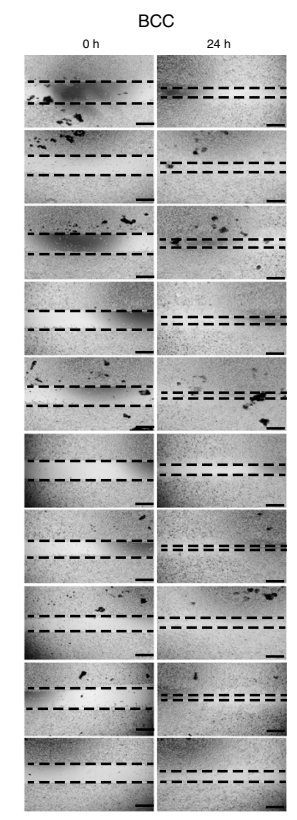

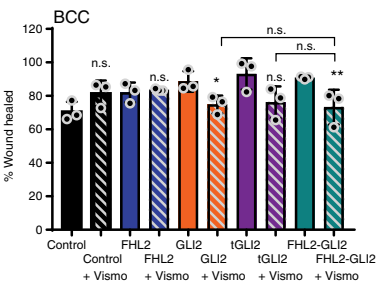

BA-Hs-FHL2-GLI2-FJ (Cat no. 801221, chr2:105984027 > chr2:121732551, 1zz pair, nt 53-90), BA-Hs-FHL2-GLI2-FJ-O1 (Cat no. 801231, chr2:106002818 > chr2:121729517, 1zz pair, nt 57-94) (Supplementary Table 3). The BaseScope Reagent Kit (Advanced Cell Diagnostics) was used and FFPE tissue sections were prepared according to the manufacturer's instructions. Each sample was quality controlled for RNA integrity with a $1 \mathrm{zz}$ probe specific to the housekeeping gene $P P I B$. Negative control background staining was evaluated using a $1 \mathrm{zz}$ probe specific to the bacterial $d a p B$ gene.
Reverse transcription PCR (RT-PCR) for fusion validation. Total RNA was reverse-transcribed using SuperScript VILO Master Mix (Life Technologies; Thermo Fisher Scientific), according to the manufacturer's instructions. PCR amplification of $10 \mathrm{ng}$ of cDNA was performed using specific primer sets designed based for each fusion gene and each breakpoint (Supplementary Table 3) ${ }^{51}$. PCR fragments were purified (ExoSAP-IT, Affymetrix) and sequenced on an ABI 3730 capillary sequencer using the ABI BigDye Terminator chemistry (v3.1, Life

Technologies) according to the manufacturer's protocol. Sequences of the forward 
Fig. 6 Inhibition of the Sonic Hedgehog pathway by Vismodegib reverses oncogenic properties. a Cell titer blue proliferation assay of immortalized mesenchymal stem cells (MSCs), HEK-293, medulloblastoma (DAOY) and human basal cell carcinoma (BCC) cells stably expressing empty vector (control), wild-type FHL2 (FHL2), wild-type GLI2 (GLI2), truncated GLI2 (tGLI2) or FHL2-GLI2 treated with 250 nM Vismodegib or vehicle control (DMSO). b Representative images of colony formation assay of MSC, HEK-293, DAOY, and BCC cells stably expressing control, FHL2, GLI2, tGLI2, or FHL2-GLI2 treated with Vismodegib $500 \mathrm{nM}$ or vehicle control (DMSO). Scale bars, $5 \mathrm{~mm}$. Quantification of the number of colonies/well compared to control (bottom). c Wound healing assay of MSC, HEK-293, DAOY, and BCC cells stably expressing control, FHL2, GLI2, tGLI2, or FHL2-GLI2 treated with $250 \mathrm{nM}$ Vismodegib or vehicle control (DMSO). The migratory effect/wound area was assessed at 0 and $24 \mathrm{~h}$ and quantified compared to DMSO (bottom). Vismo, Vismodegib. Scale bars, $500 \mu \mathrm{m}$. In a-c, data are representative of at least three independent experiments. Error bars, s.d. of mean; n.s., not significant; ${ }^{\star} P<0.05,{ }^{\star \star} P<0.01,{ }^{\star \star \star} P<0.001$; two-tailed unpaired $t$-test.

and reverse strands were analyzed using MacVector software (MacVector, Inc). All analyses were performed in duplicate.

GLI2 and Ki67 immunohistochemistry. Representative $4 \mu \mathrm{m}$ FFPE SST tissue sections on Superfrost Plus slides (Thermo Fisher Scientific) were subjected to antigen retrieval using Epitope Retrieval 1 (ER1) for 30 min. Double immunohistochemical staining for GLI2 and Ki67 was performed using the ChromoPlex 1 Dual Detection for BOND kit (Leica, DS9477) with a mouse monoclonal antibody against GLI2 (clone OTl1G1, TA804601, Origene) at a dilution of 1:500 $(2 \mu \mathrm{g} / \mathrm{ml})$ stained with red chromogen and a mouse monoclonal against Ki67 (MIB-1, Dako) at a dilution of 1:400 $(46 \mu \mathrm{g} / \mathrm{ml})$ stained with $3,3^{\prime}$-diaminobenzidine tetrahydrochloride (DAB) for $30 \mathrm{~min}$ and visualized on a Leica Bond-III autostainer (Leica, Buffalo Grove, III). Negative controls (omission of the primary antibody and substitution of the primary antibody with IgG-matched control) and positive controls (formalin-fixed, paraffin-embedded pellets of cell lines stably expressing GLI2) were included in each slide run, as described ${ }^{46}$.

Cell lines. Immortalized mesenchymal stem cells (MSCs; ASC52telo, ATCC), HEK-293 cells (ATCC), DAOY medulloblastoma cells (ATCC), and Human Basal Cell Carcinoma (BCC; CELPROGEN) primary cells were authenticated using short tandem repeat profiling at MSKCC IGO and tested for mycoplasma using the PCRbased Universal Mycoplasma Detection kit (ATCC). MSCs were cultured in Mesenchymal Stem Cell Growth Medium (Lonza). HEK-293 cells were cultured in Dulbecco's modified Eagle's medium (DMEM) high glucose supplemented with $10 \%$ fetal bovine serum (FBS) and 1\% penicillin/streptomycin. DAOY cells were cultured in Eagle's Minimum Essential Medium (EMEM) supplemented with $10 \%$ fetal bovine serum (FBS) and 1\% penicillin/streptomycin. BCC cells were cultured in Human Basal Cell Carcinoma Cell Line Complete Media (CELPROGEN). All cell lines were maintained in a $5 \% \mathrm{CO}_{2}$ atmosphere at $37^{\circ} \mathrm{C}$.

Generation of stable cell lines. Human wild-type FHL2 (EX-M0686-Lv102), wild-type GLI2 (EX-Y4001-Lv102), truncated GLI2 lacking the N-terminal domain constructed from GLI2 (EX-Y4001-Lv102), FHL2-GLI2 (CS-Y4001-Lv102-01) ORF cDNA clones with N-terminal FLAG tag were purchased from GeneCopoeia. Individual FLAG-tagged cDNAs were amplified and cloned into pCDH-CMVMCS-EF1a-GFP-T2A-Puro cDNA Dual Promoter Cloning and Expression Lentivector (CD513B-1, System Biosciences) (primers, see Supplementary Table 5). Empty vector (EV) was used as a control. Lentiviral particles were produced in MSKCC's Gene Editing and Screening Core Facility. MSC, HEK-293, DAOY and BCC cells were infected for $24 \mathrm{~h}$ and then selected for 14 days in puromycin $(2 \mu \mathrm{g} /$ $\mathrm{ml}$; ThermoFisher Scientific). Transduction efficiency was confirmed by quantitative RT-PCR and western blot.

FHL2-GLI2 mutagenesis. R338A-K339A mutations were introduced into the FHL2-GLI2 sequence using the Q5 Mutagenesis Kit (New England Biolabs) following the manufacturer's instructions and transformed in NEB Stable E.Coli to avoid unspecific recombination of the plasmid. The mutagenesis primers were designed using the NEBaseChanger (Forward: 5-CAGCTCTCTCgcggcgCATGTG AAAAC-3; Reverse: 5-GGGTCTGTGTATCTCTTG-3) and the mutations validated using Sanger sequencing (primer: 5-ggagcagaagccttcaa-3)

Luciferase assays. Stable HEK-293, DAOY, and BCC cell lines expressing empty vector, wild-type FHL2, wild-type GLI2, truncated GLI2 (tGLI2), and FHL2-GLI2 were plated in 96 well plates $(25,000$ cells/well) and transfected with lipofectamine 3000 (Thermo Fisher Scientific) following the manufacturer's instructions. Gli-Luc reporter pGL4[luc2P/Gli-RE/Hygro] (90 ng; Promega) and the transfection efficiency control Renilla-SV40 (10 ng; Promega) were added, incubated for $48 \mathrm{~h}$ and the luciferase levels assessed using the Dual luciferase kit (Dual luciferase kit; Promega) and read on the Glomax96 luminometer following the manufacturer's recommendations. SUFU overexpressing plasmid (NM_016169, Human Tagged ORF Clone from Origene) was co-transfected where indicated. HEK-293 cells were transiently co-transfected with CD513B1_FHL2-GLI2 or CD513B1_FHL2GLI2_Mutated or CD513B1 empty vector were indicated.
Quantitative RT-PCR. Total RNA was extracted from cell lines using the RNeasy Mini Kit (Qiagen) and tumors using RNeasy FFPE kit (Qiagen) and reversetranscribed into cDNA using SuperScript VILO Master Mix (ThermoFisher) according to the manufacturer's instructions. Quantitative TaqMan RT-PCR was performed for FHL2 (Hs00991866), GLI2 (Hs00171790), CALB2 (Hs00242372), GLI1 (Hs00171790), PTCH1 (Hs00181117), FOXL2 (Hs00846401), and CCND1 (Hs00765553_m1) using the StepOnePlus Real-Time PCR System (Applied Biosystems; ThermoFisher). All experiments were performed in triplicate, and expression data were normalized to GAPDH (Hs02786624), as described ${ }^{68}$.

Crosslinked chromatin immunoprecipitation (ChIP). The ChIP assay was performed as described ${ }^{69}$. Briefly, ten million MSC and HEK-293 cells expressing either empty vector or FHL2-GLI2 were used per ChIP. Chromatin was crosslinked by the addition of $1 \%$ formaldehyde (Sigma-Aldrich), and DNA was sheared to 200-500 bp fragments by sonication (Bioruptor, Diagenode). For ChIP, $50 \mu \mathrm{l}$ ANTI-FLAG M2 Affinity Gel (Sigma) were added and incubated at $4{ }^{\circ} \mathrm{C}$ overnight. Beads were incubated with elution buffer $(50 \mathrm{mM}$ Tris $\mathrm{pH} 8.0,10 \mathrm{mM}$ EDTA, $1 \%$ SDS, $0.1 \mathrm{M} \mathrm{NaHCO}_{3}$, and $250 \mathrm{mM} \mathrm{NaCl}$ ) at $65^{\circ} \mathrm{C}$ overnight to elute the immune complexes. ChIP and input DNA were purified using a QIAquick PCR purification kit (Qiagen). qPCR was performed using PowerUp SYBR Green Master Mix (Applied Biosystems). Primers for GLI1, PTCH1, and MYOD1 are listed in Supplementary Table 6.

Immunoprecipitation. For SUFU immunoprecipitation, 10 million HEK-293 cells were treated with MG-132 $10 \mu \mathrm{M}$ for $6 \mathrm{~h}$. Protein was extracted with IP buffer ( $1 \%$ NP-40, 50 mM Tris- $\mathrm{HCl}$ pH 8.0, $150 \mathrm{mM} \mathrm{NaCl}+$ Halt Protease Inhibitor Cocktail Thermo Fisher Scientific), and $500 \mu \mathrm{g}$ of protein lysate were preincubated for $1 \mathrm{~h}$ at $4{ }^{\circ} \mathrm{C}$ with $4 \mu \mathrm{l}$ of SUFU antibody (C81H7; Rabbit mAb \#2522; Cell Signaling Technology) in $500 \mu \mathrm{l}$ IP buffer. Twenty five microliter of Protein G Dynabeads (Thermo Fisher Scientific) were added and incubated overnight at $4{ }^{\circ} \mathrm{C}$. After washing the beads 3 times in IP Buffer and resuspension in $20 \mu \mathrm{l}$ of $1 \times$ NuPAGE LDS buffer, the beads were heated at $90{ }^{\circ} \mathrm{C}$ for $10 \mathrm{~min}$ and the supernatant was recovered and run on a western blot as described below. The membrane was blotted with anti-FLAG M2, SUFU and tubulin antibodies (1:1000). Total protein extractions were also tested as loading control.

Western blotting. Standard western blotting was conducted ${ }^{70}$. Cell pellets were solubilized in RIPA buffer with 5\% Halt Protease and Phosphatase Inhibitor Cocktail and 2\% $0.5 \mathrm{mM}$ EDTA (all Thermo Fisher Scientific). Extracts were centrifuged and the supernatant was then recovered for SDS-PAGE. Primary antibodies against calretinin (Santa Cruz, SC-365956, 1:500), PTCH1 (Abcam, ab53715, 1:1000), GLI1 (Abcam, ab134906, 1:500), GLI2 (Origene, TA804601, 1:500), Cyclin D1 (Cell Signaling, 92G2, \#2978, 1:1000), FLAG M2 (Sigma, F1804, 1:1000), SUFU (Cell Signaling, \#2522 s, 1:1000), and tubulin (Cell Signaling, DM1A, 1:1000). Conjugated secondary antibodies anti-rabbit (LI-COR, 926-68073, $1: 10,000)$ and anti-mouse (LI-COR, 926-32212, 1:10,000) were used and detected using the Odyssey Infrared Imaging System (LI-COR Biosciences), as described (Supplementary Figs. 10-13 $)^{70}$. Quantification and analysis were performed using ImageJ. Experiments were performed in triplicate.

Proliferation assay. Cells were seeded in 96 -well plates (1000 cells/well; $n=6$ for each cell line and condition). Proliferation rate was assessed using the Cell TiterBlue Cell Viability Assay (Promega) ${ }^{46,50}$. Absorbance was detected with $560 \mathrm{~nm}$ excitation and $590 \mathrm{~nm}$ emission using a Victor X4 Multimode Plate Reader (PerkinElmer). Experiments were performed in triplicate.

Scratch wound healing assay. Cells were seeded in 24 -well plates at $90-95 \%$ confluence. On the following day, a scratch was made on the cell monolayer using a $1 \mathrm{ml}$ pipette tip across the center of the well. Phase-contrast images were obtained at 0 and $24 \mathrm{~h}$ following scratch wounding, using an EVOS XL Core Microscope (ThermoFisher Scientific), the wound area was measured using ImageJ, and the data analyzed to determine percent of wound closure, as described ${ }^{50}$. Experiments for each condition were performed in triplicate in at least 3 independent experiments. 
Colony formation assay. Cells were seeded in 6-well plates (500-100 cells/well). After 10 days, cells were fixed and stained using the sulforhodamine B (SRB) protocol, as described 46,50 . In brief, cells were fixed at $4{ }^{\circ} \mathrm{C}$ for $1 \mathrm{~h}$ using Trichloroacetic acid (TCA) (Sigma), washed then stained on a rocking platform for 30 min with $0.04 \%$ SRB in $1 \%$ acetic acid. Plates were imaged using Zeiss Observer $\mathrm{Z} 1, \times 5 / 0.5 \mathrm{NA}$ objective and Hamamatsu Flash V3 cMOS camera. Motorized stage was calibrated to tile-image the entirety of each well. Stitched images were analyzed in ImageJ/FIJI using customized macro script. Gaussian and median filters were used to blur the image before colonies were detected by intensity threshold. Touching colonies were separated using Watershed algorithm. The number of colonies and the area of each were analyzed. Quantification of the number of colonies per well and colony size was performed using ImageJ. Experiments were performed in triplicate.

Immunofluorescence. MSCs HEK-293 cells were grown on glass coverslips and fixed with $4 \%$ formaldehyde (ThermoFisher Scientific), blocked in $10 \%$ normal Goat Serum (Vector Laboratories, S-1000) then incubated with primary antibody against Calretinin (Santa Cruz, sc-565956, 1:100) followed by Alexa Fluorconjugated secondary antibody Alexa 647 goat anti-mouse (Life Technologies, A21236, 1:500). For GLI2 nuclear localization studies, HEK-293 cells in slide chambers were fixed with ice cold solution 1:1 acetone:methanol for $15 \mathrm{~min}$ at $-20^{\circ} \mathrm{C}$. After washing 3 times with PBS the samples were blocked with blocking solution (PBS; $5 \%$ goat serum). The samples were incubated overnight at $4{ }^{\circ} \mathrm{C}$ with anti-FLAG M2 antibody (Sigma, F-1804, 1:200 in PBS/1\% BSA), followed by Alexa 647 tagged secondary goat anti-mouse antibody (Life Technologies, A21236, 1:500). Slides were mounted using Gold Prolong antifade reagent containing DAPI (ThermoFisher Scientific, P36930). Fluorescent images were acquired at MSKCC's Molecular Cytology Core using a TCS SP5 upright Confocal microscope (Leica Microsystems), with a $\times 63 / 1.40$ oil objective, HyD hybrid detectors, and the Leica Application Suite Advanced Fluorescence (LASAF) acquisition software (Leica Microsystems). DAPI and Alexa647 were excited with $405 \mathrm{~nm}$ and $650 \mathrm{~nm}$ lasers, respectively, and imaged in the range of $410-460 \mathrm{~nm}$ and $660-700 \mathrm{~nm}$, respectively. Linear LUT was used at full range. Images were acquired as LIF files (Leica image file format; Leica Microsystems) at a resolution of $1024 \times 1024$ pixels, a scaling of $0.12 \mathrm{micron} /$ pixel, and an 8-bit depth and processed using Fiji and ImageJ. No post-acquisition processing was performed, besides minor adjustments of brightness and contrast, applied equally to all images. ImageJ software was used to quantify the number of pixels per cell analyzing at least five representative images $(\times 40$ field) for each condition. Calretinin images were analyzed using ImageJ/FIJI with a custom macro. The number of cells within each image were counted by segmenting the DAPI channels. After setting an appropriate threshold, total intensity of calretinin was measured for the image then normalized to the number of cells. Experiments for each condition were performed in triplicate in at least 3 independent experiments.

Inhibitors. The smoothened inhibitors Cyclopamine (Selleckchem, S1146), Vismodegib (Cellagen tech, C4044-5) and the GLI inhibitor GANT61 (Tocris, 3191) were resuspended in DMSO and used in in vitro proliferation, colony formation and scratch wound healing assays at 5-10 $\mu \mathrm{M}, 250-500 \mathrm{nM}$, and 5-10 $\mu \mathrm{M}$, respectively. Experiments were performed in triplicate.

Statistical analysis. Statistical analysis was performed using Prism 7 (GraphPad). Student's two-tailed $t$-test was employed for the comparison of means in parametric data. The heteroscedasticity was assessed for each comparison, and homoscedastic or heteroscedastic $t$-tests were employed as appropriate, as described $^{50}$. Wilcoxon rank test was used for the comparison of the SHH enrichment scores. A $P$ value of $<0.05$ was considered significant.

Reporting summary. Further information on research design is available in the Nature Research Reporting Summary linked to this article.

\section{Data availability}

RNA-sequencing data have been deposited in Sequence Read Archive (SRA) with accession code PRJNA540984. WES and targeted sequencing data are available via cBioPortal for Cancer Genomics (www.cbioportal.org). The remaining data are available in the Article or Supplementary Material.

Received: 15 May 2019; Accepted: 26 November 2019; Published online: 02 January 2020

\section{References}

1. McCluggage, W. G., Kiyokawa, T., Staats, P. N. \& Young, R. H. in WHO Classification of Tumours of Female Reproductive Organs (eds Kurman, R. J.,
Carcangiu, M.-L., Herrington, C. S. \& Young, R. H.) 44-9 (International Agency for Research on Cancer, 2014).

2. Young, R. H. Ovarian sex cord-stromal tumours and their mimics. Pathology 50, 5-15 (2018).

3. Chalvardjian, A. \& Scully, R. E. Sclerosing stromal tumors of the ovary. Cancer 31, 664-670 (1973).

4. Young, R. H. Ovarian tumors and tumor-like lesions in the first three decades. Semin Diagn. Pathol. 31, 382-426 (2014).

5. Terauchi, F. et al. Sclerosing stromal tumor of the ovary with elevated CA125. J. Obstet. Gynaecol. Res 31, 432-435 (2005).

6. Khanna, M., Khanna, A. \& Manjari, M. Sclerosing stromal tumor of ovary: a case report. Case Rep. Pathol. 2012, 592836 (2012).

7. Bennett, J. A., Oliva, E. \& Young, R. H. Sclerosing stromal tumors with prominent luteinization during pregnancy: a report of 8 cases emphasizing diagnostic problems. Int. J. Gynecol. Pathol. 34, 357-362 (2015).

8. Rabban, J. T. \& Zaloudek, C. J. A practical approach to immunohistochemical diagnosis of ovarian germ cell tumours and sex cord-stromal tumours. Histopathology 62, 71-88 (2013).

9. Shah, S. P. et al. Mutation of FOXL2 in granulosa-cell tumors of the ovary. N. Engl. J. Med. 360, 2719-2729 (2009).

10. Al-Agha, O. M. et al. FOXL2 is a sensitive and specific marker for sex cordstromal tumors of the ovary. Am. J. Surg. Pathol. 35, 484-494 (2011).

11. Cancer Genome Atlas Research, N. Integrated genomic analyses of ovarian carcinoma. Nature 474, 609-615 (2011).

12. Patibandla, J. R., Fehniger, J. E., Levine, D. A. \& Jelinic, P. Small cell cancers of the female genital tract: Molecular and clinical aspects. Gynecol. Oncol. 149, 420-427 (2018).

13. Hyman, D. M., Taylor, B. S. \& Baselga, J. Implementing genome-driven oncology. Cell 168, 584-599 (2017).

14. Heravi-Moussavi, A. et al. Recurrent somatic DICER1 mutations in nonepithelial ovarian cancers. N. Engl. J. Med. 366, 234-242 (2012).

15. Conlon, N. et al. A survey of DICER1 hotspot mutations in ovarian and testicular sex cord-stromal tumors. Mod. Pathol. 28, 1603-1612 (2015).

16. Cheng, D. T. et al. Memorial sloan kettering-integrated mutation profiling of actionable cancer targets (MSK-IMPACT): a hybridization capture-based next-generation sequencing clinical assay for solid tumor molecular oncology. J. Mol. Diagn. 17, 251-264 (2015).

17. Shugay, M., Ortiz de Mendibil, I., Vizmanos, J. L. \& Novo, F. J. Oncofuse: a computational framework for the prediction of the oncogenic potential of gene fusions. Bioinformatics 29, 2539-2546 (2013).

18. Du, X. et al. The LIM-only coactivator FHL2 modulates WT1 transcriptional activity during gonadal differentiation. Biochim. Biophys. Acta 1577, 93-101 (2002).

19. Matulis, C. K. \& Mayo, K. E. The LIM domain protein FHL2 interacts with the NR5A family of nuclear receptors and CREB to activate the inhibin-alpha subunit gene in ovarian granulosa cells. Mol. Endocrinol. 26, 1278-1290 (2012).

20. Roessler, E. et al. A previously unidentified amino-terminal domain regulates transcriptional activity of wild-type and disease-associated human GLI2. Hum. Mol. Genet. 14, 2181-2188 (2005).

21. Cai, T. et al. FHL2 promotes tubular epithelial-to-mesenchymal transition through modulating beta-catenin signalling. J. Cell Mol. Med. 22, 1684-1695 (2018).

22. Pietrobono, S., Gagliardi, S. \& Stecca, B. Non-canonical hedgehog signaling pathway in cancer: activation of GLI transcription factors beyond smoothened. Front. Genet. 10, 556 (2019).

23. Pak, E. \& Segal, R. A. Hedgehog signal transduction: key players, oncogenic drivers, and cancer therapy. Dev. Cell 38, 333-344 (2016).

24. Hui, C. C. \& Angers, S. Gli proteins in development and disease. Annu Rev. Cell Dev. Biol. 27, 513-537 (2011).

25. Graham, R. P. et al. Gastroblastoma harbors a recurrent somatic MALAT1GLI1 fusion gene. Mod. Pathol. 30, 1443-1452 (2017).

26. Dahlen, A. et al. Activation of the GLI oncogene through fusion with the betaactin gene (ACTB) in a group of distinctive pericytic neoplasms: pericytoma with $\mathrm{t}(7 ; 12)$. Am. J. Pathol. 164, 1645-1653 (2004).

27. Spans, L. et al. Recurrent MALAT1-GLI1 oncogenic fusion and GLI1 upregulation define a subset of plexiform fibromyxoma. J. Pathol. 239, 335-343 (2016).

28. Hu, X. et al. TumorFusions: an integrative resource for cancer-associated transcript fusions. Nucleic Acids Res. 46, D1144-D1149 (2018).

29. Raffel, C. et al. Sporadic medulloblastomas contain PTCH mutations. Cancer Res. 57, 842-845 (1997).

30. Xie, J. et al. Activating Smoothened mutations in sporadic basal-cell carcinoma. Nature 391, 90-92 (1998).

31. Cao, Q. J., Jones, J. G. \& Li, M. Expression of calretinin in human ovary, testis, and ovarian sex cord-stromal tumors. Int. J. Gynecol. Pathol. 20, 346-352 (2001). 
32. Movahedi-Lankarani, S. \& Kurman, R. J. Calretinin, a more sensitive but less specific marker than alpha-inhibin for ovarian sex cord-stromal neoplasms: an immunohistochemical study of 215 cases. Am. J. Surg. Pathol. 26, 1477-1483 (2002).

33. Infante, P. et al. Gli1/DNA interaction is a druggable target for Hedgehogdependent tumors. EMBO J. 34, 200-217 (2015).

34. Han, Y., Shi, Q. \& Jiang, J. Multisite interaction with Sufu regulates Ci/Gli activity through distinct mechanisms in Hh signal transduction. Proc. Natl Acad. Sci. USA 112, 6383-6388 (2015).

35. Yan, J. et al. Transcription factor binding in human cells occurs in dense clusters formed around cohesin anchor sites. Cell 154, 801-813 (2013).

36. Coquenlorge, $\mathrm{S}$. et al. GLI2 modulated by SUFU and SPOP induces intestinal stem cell niche signals in development and tumorigenesis. Cell Rep. 27, 3006-3018 (2019). e3004.

37. Rimkus, T. K., Carpenter, R. L., Qasem, S., Chan, M. \& Lo, H. W. Targeting the sonic hedgehog signaling pathway: review of smoothened and GLI inhibitors. Cancers 8, 22 (2016).

38. Kasper, M., Regl, G., Frischauf, A. M. \& Aberger, F. GLI transcription factors: mediators of oncogenic Hedgehog signalling. Eur. J. Cancer 42, 437-445 (2006).

39. Sasaki, H., Nishizaki, Y., Hui, C., Nakafuku, M. \& Kondoh, H. Regulation of Gli2 and Gli3 activities by an amino-terminal repression domain: implication of Gli2 and Gli3 as primary mediators of Shh signaling. Development 126, 3915-3924 (1999).

40. Wijgerde, M., Ooms, M., Hoogerbrugge, J. W. \& Grootegoed, J. A. Hedgehog signaling in mouse ovary: Indian hedgehog and desert hedgehog from granulosa cells induce target gene expression in developing theca cells. Endocrinology 146, 3558-3566 (2005).

41. Richards, J. S., Ren, Y. A., Candelaria, N., Adams, J. E. \& Rajkovic, A. Ovarian follicular theca cell recruitment, differentiation, and impact on fertility: 2017 update. Endocr. Rev. 39, 1-20 (2018).

42. Mertens, F., Johansson, B., Fioretos, T. \& Mitelman, F. The emerging complexity of gene fusions in cancer. Nat. Rev. Cancer 15, 371-381 (2015)

43. Persson, M. et al. Recurrent fusion of MYB and NFIB transcription factor genes in carcinomas of the breast and head and neck. Proc. Natl Acad. Sci. USA 106, 18740-18744 (2009).

44. Martelotto, L. G. et al. Genomic landscape of adenoid cystic carcinoma of the breast. J. Pathol. 237, 179-189 (2015).

45. Kim, J. et al. MYBL1 rearrangements and MYB amplification in breast adenoid cystic carcinomas lacking the MYB-NFIB fusion gene. J. Pathol. 244, 143-150 (2018).

46. Weinreb, I. et al. Hotspot activating PRKD1 somatic mutations in polymorphous low-grade adenocarcinomas of the salivary glands. Nat. Genet. 46, 1166-1169 (2014).

47. Weinreb, I. et al. Novel PRKD gene rearrangements and variant fusions in cribriform adenocarcinoma of salivary gland origin. Genes Chromosomes Cancer 53, 845-856 (2014).

48. Tan, I. L. et al. Lateral cerebellum is preferentially sensitive to high sonic hedgehog signaling and medulloblastoma formation. Proc. Natl Acad. Sci. USA 115, 3392-3397 (2018).

49. Epstein, E. H. Basal cell carcinomas: attack of the hedgehog. Nat. Rev. Cancer 8, 743-754 (2008).

50. Pareja, F. et al. Loss-of-function mutations in ATP6AP1 and ATP6AP2 in granular cell tumors. Nat. Commun. 9, 3533 (2018).

51. Pareja, F. et al. The genomic landscape of mucinous breast cancer. J. Natl Cancer Inst. https://doi.org/10.1093/jnci/djy216 (2019). [Epub ahead of print].

52. Chang, M. T. et al. Accelerating discovery of functional mutant alleles in cancer. Cancer Disco. 8, 174-183 (2018).

53. Shen, R. \& Seshan, V. E. FACETS: allele-specific copy number and clonal heterogeneity analysis tool for high-throughput DNA sequencing. Nucleic Acids Res. 44, e131 (2016).

54. Carter, S. L. et al. Absolute quantification of somatic DNA alterations in human cancer. Nat. Biotechnol. 30, 413-421 (2012).

55. Basturk, O. et al. Sclerosing epithelioid mesenchymal neoplasm of the pancreas-a proposed new entity. Mod. Pathol. https://doi.org/10.1038/ s41379-019-0334-5 (2019). [Epub ahead of print].

56. Zhang, J. et al. INTEGRATE: gene fusion discovery using whole genome and transcriptome data. Genome Res. 26, 108-118 (2015).

57. McPherson, A. et al. deFuse: an algorithm for gene fusion discovery in tumor RNA-Seq data. PLoS Comput. Biol. 7, e1001138 (2011)

58. Edgren, $\mathrm{H}$. et al. Identification of fusion genes in breast cancer by paired-end RNA-sequencing. Genome Biol. 12, R6 (2011).

59. Cancer Genome Atlas, N. Comprehensive molecular portraits of human breast tumours. Nature 490, 61-70 (2012).

60. Robinson, M. D., McCarthy, D. J. \& Smyth, G. K. edgeR: a Bioconductor package for differential expression analysis of digital gene expression data. Bioinformatics 26, 139-140 (2010).
61. Robinson, M. D. \& Smyth, G. K. Moderated statistical tests for assessing differences in tag abundance. Bioinformatics 23, 2881-2887 (2007).

62. McCarthy, D. J., Chen, Y. \& Smyth, G. K. Differential expression analysis of multifactor RNA-Seq experiments with respect to biological variation. Nucleic Acids Res. 40, 4288-4297 (2012).

63. Benjamini, Y., Drai, D., Elmer, G., Kafkafi, N. \& Golani, I. Controlling the false discovery rate in behavior genetics research. Behav. Brain Res. 125, 279-284 (2001).

64. Fusco, N. et al. Resolving quandaries: basaloid adenoid cystic carcinoma or breast cylindroma? The role of massively parallel sequencing. Histopathology 68, 262-271 (2016)

65. Wang, H. et al. NanoStringDiff: a novel statistical method for differential expression analysis based on NanoString nCounter data. Nucleic Acids Res. 44, e151 (2016).

66. Barbie, D. A. et al. Systematic RNA interference reveals that oncogenic KRASdriven cancers require TBK1. Nature 462, 108-112 (2009).

67. Wang, F. et al. RNAscope: a novel in situ RNA analysis platform for formalinfixed, paraffin-embedded tissues. J. Mol. Diagn. 14, 22-29 (2012).

68. Geyer, F. C. et al. Recurrent hotspot mutations in HRAS Q61 and PI3K-AKT pathway genes as drivers of breast adenomyoepitheliomas. Nat. Commun. 9 , 1816 (2018)

69. Mohammad, F. et al. EZH2 is a potential therapeutic target for H3K27Mmutant pediatric gliomas. Nat. Med. 23, 483-492 (2017).

70. Weigelt, B., Warne, P. H. \& Downward, J. PIK3CA mutation, but not PTEN loss of function, determines the sensitivity of breast cancer cells to mTOR inhibitory drugs. Oncogene 30, 3222-3233 (2011).

\section{Acknowledgements}

We thank the Integrated Genomics Operation, Gene Editing and Screening, Molecular Cytogenetics, and Molecular Cytology Core Facilities at MSK. Research reported in this publication was funded in part by a Cancer Center Support Grant of the National Institutes of Health/National Cancer Institute (grant no. P30CA008748). J.S.R.-F. is funded in part by the Breast Cancer Research Foundation, B.W. by Cycle for Survival, C.S. by a Fundação para a Ciência e Tecnologia grant (SFRH/BDE/110544/2015). D.Z. is supported by the Ovarian Cancer Research Foundation Liz Tilberis Award, and the Department of Defense Ovarian Cancer Research Academy (OC150111). K.H. is funded in part by the Independent Research Fund Denmark (7016-00067) and through a center grant from the NNF to the NNF Center for Stem Cell Biology (NNF17CC0027852). Some of the material was obtained from the archives of the late Dr. Robert E Scully.

\section{Author contributions}

B.W., R.B. and J.S.R.-F. conceived the study. R.B., B.W., W.R.S. and E.O. provided tissue samples. R.A.S., R.B., F.P., A.P.M.S., J.S.R.-F. and E.O. performed histopathologic review. Sample processing and nucleic acid extractions were performed by A.D.C.P., R.B., E.M.D.S, R.G.-M., C.W.A., C.S. and A.S. A.A.J. performed immunohistochemistry. R.G.-M. performed RNA-sequencing analysis for the identification and validation of fusion genes; A.D.C.P., L.F. and P.S. performed bioinformatics analyses, which were coordinated by B.W. and J.S.R.-F. Functional experiments were performed by S.H.K., T.B., H.D., F.P., E.M.D.S., Z.S., C.G.S., Y.B., R.B., C.S. and A.L. S.F. performed imaging quantification. S.H.K., A.D.C.P., T.B., H.D., R.B., F.P., Z.S., K.H., D.Z., N.R.A.-R., J.S.R.-F. and B.W. interpreted the results. S.H.K., A.D.C.P., T.B., F.P., J.S.R.-F. and B.W wrote the first draft. All authors read, edited, and approved the final paper.

\section{Competing interests}

D.Z. reports consulting fees from Merck, Synlogic Therapeutics, Tizona Therapeutics, and Tesaro, outside the submitted work. D.Z. has a patent for use of Newcastle Disease Virus for cancer therapy, outside the submitted work. J.S. Reis-Filho reports personal/ consultancy fees from VolitionRx, Page.AI, Invicro, Roche, Genentech, Ventana, Gold man Sachs and REPARE Therapeutics, outside the submitted work. The remaining authors declare no competing interests.

\section{Additional information}

Supplementary information is available for this paper at https://doi.org/10.1038/s41467 019-13806-x.

Correspondence and requests for materials should be addressed to E.O. or B.W.

Peer review information Nature Communications thanks Kris Ann Schultz and the other, anonymous, reviewer(s) for their contribution to the peer review of this work

Reprints and permission information is available at http://www.nature.com/reprints

Publisher's note Springer Nature remains neutral with regard to jurisdictional claims in published maps and institutional affiliations. 
(c) (i) Open Access This article is licensed under a Creative Commons Attribution 4.0 International License, which permits use, sharing, adaptation, distribution and reproduction in any medium or format, as long as you give appropriate credit to the original author(s) and the source, provide a link to the Creative Commons license, and indicate if changes were made. The images or other third party material in this article are included in the article's Creative Commons license, unless indicated otherwise in a credit line to the material. If material is not included in the article's Creative Commons license and your intended use is not permitted by statutory regulation or exceeds the permitted use, you will need to obtain permission directly from the copyright holder. To view a copy of this license, visit http://creativecommons.org/ licenses/by/4.0/.

(C) The Author(s) 2020 